\title{
A single-station method for the detection, classification and location of fin whale calls using ocean-bottom seismic stations
}

\author{
Luis Matias ${ }^{1, a)}$ and Danielle Harris ${ }^{2}$ \\ ${ }_{1}^{1}$ Instituto Dom Luiz, Faculdade de Ciências, Universidade de Lisboa, Ed. C8, piso 3, 1749-016 Lisbon, \\ Portugal \\ ${ }^{2}$ Centre for Research into Ecological and Environmental Modelling, The Observatory, Buchanan Gardens, \\ University of St. Andrews, St. Andrews, Fife KY16 9LZ, United Kingdom
}

(Received 26 July 2014; revised 17 May 2015; accepted 7 June 2015; published online 24 July 2015)

\begin{abstract}
Passive seismic monitoring in the oceans uses long-term deployments of Ocean Bottom Seismometers (OBSs). An OBS usually records the three components of ground motion and pressure, typically at $100 \mathrm{~Hz}$. This makes the OBS an ideal tool to investigate fin and blue whales that vocalize at frequencies below $45 \mathrm{~Hz}$. Previous applications of OBS data to locate whale calls have relied on single channel analyses that disregard the information that is conveyed by the horizontal seismic channels. Recently, Harris, Matias, Thomas, Harwood, and Geissler [J. Acoust. Soc. Am. 134, 3522-3535 (2013)] presented a method that used all four channels recorded by one OBS to derive the range and azimuth of fin whale calls. In this work, the detection, classification, and ranging of calls using this four-channel method were further investigated, focusing on methods to increase the accuracy of range estimates to direct path arrivals. Corrections to account for the influences of the sound speed in the water layer and the velocity structure in the top strata of the seabed were considered. The single station method discussed here is best implemented when OBSs have been deployed in deep water on top of seabed strata with low $P$-wave velocity. These conditions maximize the ability to detect and estimate ranges to fin whale calls.
\end{abstract}

(C) 2015 Acoustical Society of America. [http://dx.doi.org/10.1121/1.4922706]

[AMT]

Pages: 504-520

\section{INTRODUCTION}

Monitoring marine mammals using static acoustic sensors is a common practice but a costly one, particularly in the deep ocean. For this reason, datasets acquired for other purposes, such as seismic monitoring, are extremely useful and valuable. Ocean bottom seismometers (OBSs) usually sample data at 50 to $100 \mathrm{~Hz}$, so are suitable for recording low frequency calls of large baleen whales such as blue (Balaenoptera musculus sp.) and fin whales (Balaenoptera physalus) (McDonald et al., 1995). The large number of past, ongoing, and planned passive seismic experiments, along with the increasing number of available OBSs, provide a substantial dataset has been underutilized for marine mammal studies, until recently (e.g., Rebull et al., 2006; Wilcock, 2012).

It is possible to use OBSs for the location of sound sources in the ocean, in addition to their primary function of identifying and localizing seismic events. A number of different localization methods have been used to localize and track blue and fin whale with OBSs. Multipath differences can be used for ranging purposes from single OBSs and multi-station techniques can also be used for source location (e.g., Rebull et al., 2006; Wilcock, 2012; Weirathmueller and Wilcock, 2013). However, multi-station methods require that sensors are located close together (typically a maximum

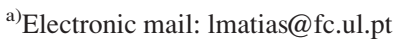

spacing of 10 to $15 \mathrm{~km}$ ) and multipath ranging with a single sensor does not allow the two-dimensional (2D) location of the sound source to be estimated. In addition, location of sound sources in the ocean is more complex than in traditional crustal seismology due to the effects of oceanic sound velocity profiles on sound propagation.

Recently, it has been shown that an alternative single station method can be used for fin whale call localization at close ranges and with sufficient precision to be used in animal density estimates (Harris et al., 2013). The basics of this method and its application to the localization of fin whale calls are presented in Harris et al. (2013). The main features of the method are (i) the method only works for direct path vocalizations from sources that are inside a critical range. The critical range is a function of the sound speed in the water column and the $P$-wave velocity in the top strata of the seabed; (ii) the seismic signal recorded by a threecomponent seismometer is used to estimate the apparent emergence angle of the $P$-wave in the seabed by applying the polarization analysis method of Roberts et al. (1989); (iii) the top seabed strata properties (including $S$-wave properties) are then used to convert this apparent angle to the true incident angle of the acoustic sound wave at the seafloor; (iv) this latter angle, and water depth, are finally used to estimate the location of the sound source considered to be close to the sea surface.

Here, the presentation of this single sensor method (SSM) is expanded, detailing the methodologies used for detection and classification of fin whale vocalizations and 
estimating ranges to calls. The effects that realistic sound propagation in both the water column and the top seabed strata have on the estimated ranges are also discussed.

In Harris et al. (2013) the feasibility of the SSM to estimate true ranges to sound sources close to the surface was validated by the analysis of airgun shots recorded in the Gulf of Cadiz during the NEAREST (2012) passive seismic experiment. Here, the same dataset is used to examine the performance of the detection, classification, and ranging methodologies in more detail. In addition, another dataset recorded in the Azores will be used to further validate the methodology using independently located fin whale vocalizations using closely spaced sensors.

The paper is laid out as follows. Section II revisits the methodology, expanding the work presented by Harris et al. (2013) to further explore the algorithms used to detect and classify fin whale vocalizations. Section III describes the control datasets, the analyses conducted, and gives the results. Finally, Sec. IV is an overarching discussion of the results obtained, ending with some general conclusions.

\section{THE SINGLE STATION METHOD REVISITED}

There are two main motivations for further investigation of the detection, classification, and localization algorithm presented in Harris et al. (2013). First, the method will return spurious range estimates for direct path calls originating from outside the critical range or any multipathed signal. Therefore, it is important that the algorithm minimizes the probability of generating such ranges by selecting direct path calls that are strictly inside the critical range. Second, further consideration of the seabed and water properties is likely to lead to more accurate range estimation. The removal of false ranges and improving the accuracy of the remaining true ranges are both particularly important if the data are to be used for distance sampling, a standard animal density estimation approach (Buckland et al., 2001; Harris et al., 2013).

\section{A. Automatic detection of fin whale calls}

Fin whales produce a variety of sounds (Thompson et al., 1992) but the " $20-\mathrm{Hz}$ " call is the most studied fin whale vocalization and has been recorded worldwide (Sirović et al., 2007; Stafford et al., 2009; Nieukirk et al., 2012). Each call is $\sim 1 \mathrm{~s}$ in duration, sweeping downwards over a $15-30 \mathrm{~Hz}$ range. Source levels above $180 \mathrm{~dB}$ re. $1 \mu \mathrm{Pa}$ at $1 \mathrm{~m}$ have been estimated (e.g., Watkins et al., 1987). The calls are repeated in series with a regular interval of several seconds (ibid.). These characteristics make fin whale calls particularly suited to analysis by conventional seismological methods and they can be easily detected by ocean bottom sensors, including OBSs. In fact, in the Gulf of Cadiz, fin whale calls constitute the strongest source of noise as recorded by hydrophones and vertical geophones in the frequency band from 2 to $30 \mathrm{~Hz}$ (Corela, 2014).

\section{Cross correlation using a matched filter}

The $20 \mathrm{~Hz}$ call is a repetitive down sweep with an amplitude envelope displaying an asymmetric half-sinus shape (with energy slightly displaced to the front of the pulse). The amplitude and spectral band characteristics have been used by Wilcock (2012) to automatically detect fin whale calls. In contrast, the method presented here uses a matched filter method that relies on the high repeatability of the vocalizations and uses one of the loudest and highest signal to noise ratio $(\mathrm{S} / \mathrm{N})$ pulses selected from the data as the master waveform. The cross-correlation of a running time-window and the master waveform is computed and only the windows that show a normalized correlation above a pre-defined threshold are saved for further analysis.

Very early in the development of the method it was noted that the application of the matched filter using the standard expression for the normalized correlation $\left(C_{x y}^{S}\right)$ between two arbitrary time series $x_{i}$ and $y_{i}$ [Eq. (1)] resulted in a very large number of detections with good correlations but very small absolute amplitude (equivalent to very small $\mathrm{S} / \mathrm{N}$ ),

$$
C_{x y}^{S}=\frac{\sum x_{i} y_{i}}{\sqrt{\sum x_{i}^{2} \sum y_{i}^{2}}} .
$$

Fin whale vocalizations have been shown to have a high source level with some variability (e.g., Širović et al., 2007, estimated a mean source level of $189 \mathrm{~dB}$ re: $1 \mu \mathrm{Pa}$ at $1 \mathrm{~m}$ over $15-28 \mathrm{~Hz}$ with a standard deviation of $4 \mathrm{~dB}$ ). Weirathmueller et al. (2013) showed that this source level is nearly constant between geographical locations. Since sound level attenuates with distance, it can be assumed that, in general, small amplitude signals will be likely generated by farther away sources or multipathed signals. However, such signals will generate spurious range estimates and so are unsuitable for analysis by the SSM. Therefore, the normalized correlation $\left(C_{x y}^{M}\right)$ formula was modified as follows:

$$
C_{x y}^{M}=\left(\sqrt{2} \frac{\sum x_{i} y_{i}}{\sqrt{\left(\sum x_{i}^{2}\right)^{2}+\left(\sum y_{i}^{2}\right)^{2}}}\right)^{M},
$$

where $M$ is a constant to be chosen by the analyst.

If $x_{i}=y_{i}$, this correlation also gives a value of one [as Eq. (1)], but when one of the pulses is attenuated by a factor, e.g., $y_{i}=A x_{i}$, then

$$
C_{x y}^{M}=\left(\sqrt{2} \frac{1}{\sqrt{A^{2}+\frac{1}{A^{2}}}}\right)^{M} .
$$

This normalization has the effect of degrading the correlation value of signals that differ in amplitude, even if they are perfectly correlated, meaning that a given correlation threshold used in Eq. (1) becomes more selective when applied to Eq. (2) [Figs. 1(a)-1(d)]. Therefore, in order to reduce the probability of missing direct path calls within the critical range, the detection threshold for the modified 

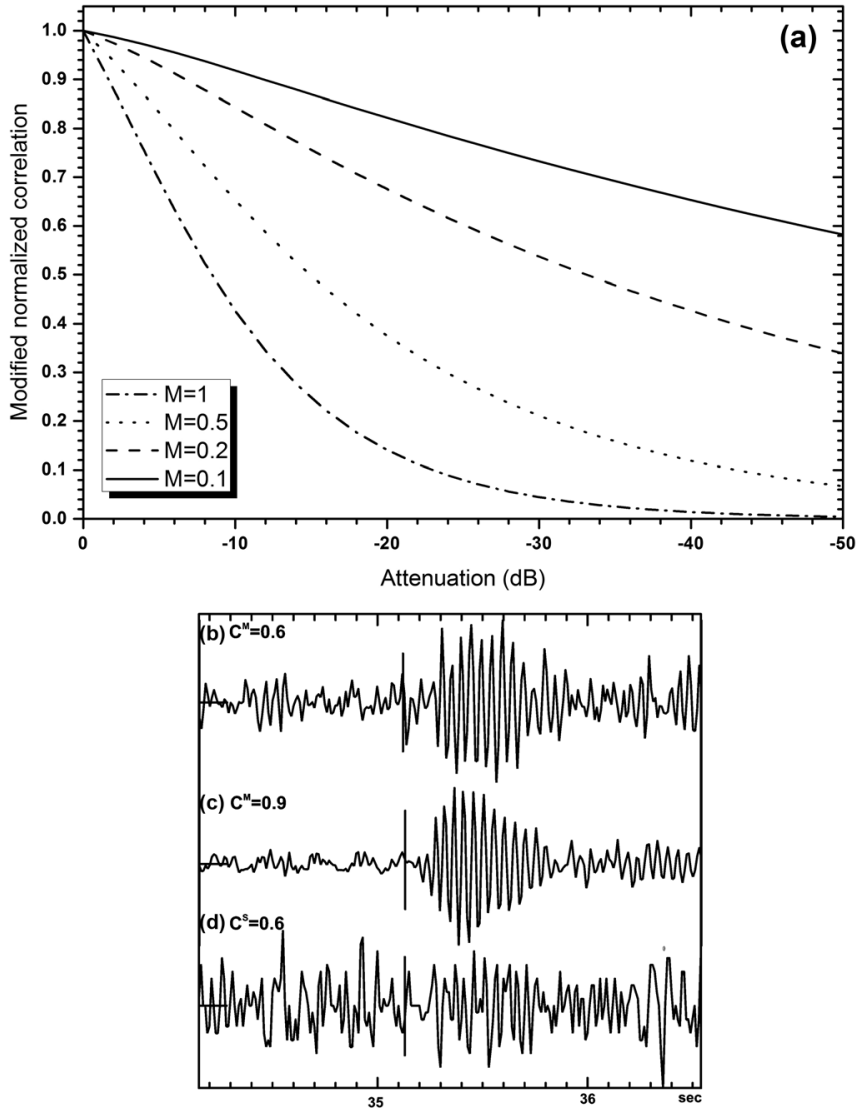

FIG. 1. (a) Attenuation that results from the application of the modified normalized cross-correlation in Eq. (2) as applied to two identical pulses that differ only in amplitude. The amplitude difference between the two signals is expressed in $\mathrm{dB}$. An example of three pulses that were detected by the matching filter method using different normalizations for the crosscorrelation. The master waveform is not shown but a nearly perfect correlated signal is presented in (c) for comparison. (b) Pulse with a modified cross-correlation of 0.6 [Eq. (2) applied with $M=0.5$ ]. (d) Pulse with a standard cross-correlation of 0.6 .

cross-correlation (hereafter referred as Cmax) may be set to a conservative value (typically 0.3 to 0.4 ). A number of diagnostic parameters are then computed for each detected pulse in order to further identify direct path calls within the critical range, which are discussed in Sec. II B. Given that the modified normalized correlation is affected by the relative amplitudes, it is important that the master signal used for the matched filter is chosen as having the largest amplitude expected in the time series to be investigated. In the two case studies investigated, the master signal was chosen as the call with the largest amplitude, confirmed by visual inspection.

\section{Rejecting multipaths-using a time buffer}

Since the SSM only works at close ranges (inside the critical range) where multipath pulses arrive a few seconds after the direct path, an additional parameter can be used to reject multipath arrivals. A "protection time buffer" $\Delta t_{P}$ is defined, inside which only the largest correlation is kept. This method prevents a very large number of unwanted detections from entering the classification procedure. However, the use of this parameter may mean that a direct path call produced shortly after another direct path call is rejected. Therefore, this parameter may be best used for the preliminary evaluation of the data when the classification parameters are being fine-tuned. When the classification procedure is defined, then this time buffer can be relaxed so that multiple whales calling simultaneously in the same area can be investigated.

\section{B. Classification of fin whale calls}

Fin whale calls classified for use with the method must not only be (1) direct path signals, but must also be (2) within a critical range. In this section, the relationship between the horizontal and vertical velocities and the influence of water column and seabed properties on the critical range are described in detail. A number of useful parameters that can be used to classify direct path fin whale calls are identified, as well as exploring whether multipath signals are likely to be misclassified as direct path signals.

\section{The coherency factor}

As explained in Harris et al. (2013) the polarization analysis of the seismic signal recorded by the threecomponent seismometer is performed using the method of Roberts et al. (1989), which uses the cross-correlation between the horizontal and vertical channels. The particle velocity that is recorded by the seismometer is defined as a vector, $\vec{v}(t)$,

$$
\vec{v}(t)=\left[\begin{array}{c}
\dot{x}(t) \\
\dot{y}(t) \\
\dot{z}(t)
\end{array}\right] .
$$

In the absence of noise or phase shifts generated by local heterogeneities and scattering, the three velocity components should be perfectly correlated with constant coefficients $a$ and $b$ that depend exclusively on the azimuth $\phi$ and apparent incident angle $i_{\text {app}}$,

$$
\begin{aligned}
& \dot{x}(t)=a P(t) \quad a=\tan \left(i_{\text {app }}\right) \sin \phi \\
& \dot{y}(t)=b P(t) \quad b=\tan \left(i_{\text {app }}\right) \cos \phi . \\
& \dot{z}(t)=P(t)
\end{aligned}
$$

In order to assess the quality of the estimated parameters $\phi$ and $i_{\text {app }}$, Roberts et al. (1989) proposed the computation of a coherency factor $C_{O}$. If there was a perfect correlation between the horizontal and vertical velocities recorded by the seismometer, then

$$
\begin{aligned}
& \dot{h}(t)=\sqrt{\dot{x}^{2}+\dot{y}^{2}=} \dot{x}(t) \sin \phi+\dot{y}(t) \cos \phi \\
& \dot{z}(t)=\frac{\dot{h}(t)}{\tan \left(i_{\text {app }}\right)}=\dot{x}(t) \frac{\sin \phi}{\tan \left(i_{\text {app }}\right)}+\dot{y}(t) \frac{\cos \phi}{\tan \left(i_{\text {app }}\right)}
\end{aligned}
$$

Equation (6) can then be used to estimate the vertical signal from the horizontal recordings

$$
\dot{z}_{\text {est }}(t)=A \dot{x}(t)+B \dot{y}(t)
$$

The predicted coherency factor $C_{O}$ is then defined by 


$$
C_{O}=1-\frac{\left\langle(\dot{z}(t)-A \dot{x}(t)-B \dot{y}(t))^{2}\right\rangle}{\langle\dot{z}, \dot{z}\rangle},
$$

where \langle\rangle means the series average. For a noiseless and ideally linearly polarized $P$-wave signal this coherency factor should be equal to one. In the other circumstances, the coherency factor is smaller than one. Therefore, using the Roberts et al. (1989) method, a measurement of azimuth and apparent incident angle may be accepted whenever the coherency factor is larger than a predefined threshold.

\section{The Zoeppritz equations and the critical range}

To locate a sound source close to the surface from the seismic signals recorded at the sea-bottom, the true emergence angle has to be estimated from the apparent emergence angle of the $P$-wave in the top seabed strata. This relationship can be obtained by solving the Zoeppritz equations that depend on the elastic properties of the water layer and seabed, considered as vertically layered isotropic media (e.g., Aki and Richards, 1980).

The use of Zoeppritz equations are a common tool used in seismic exploration for amplitude versus offset analysis (e.g., Castagna, 1993). However, this formulation is strictly valid only for plane waves propagating with an infinite frequency. It has been recognized that departures from amplitudes derived by the Zoeppritz equations do occur when band limited signals from point sources are considered (e.g., Favretto-Cristini et al., 2007). We will begin by assuming that the conditions at the seafloor where OBS recordings are obtained meet the assumptions required by the Zoeppritz equations. Departures from these conditions will be addressed in Sec. IV.

Taking $20 \mathrm{~Hz}$ as the reference frequency for the fin whale calls and $2000 \mathrm{~m} / \mathrm{s}$ as a typical value for the $P$-wave velocity in the shallow rocks, then the wavelength of the acoustic signal in the seabed is typically $100 \mathrm{~m}$. The radius of the first Fresnel Zone (where reflected energy is reflected in phase) is then $\sim 25 \mathrm{~m}$. This implies that estimating the amplitude of the recorded signals requires knowledge of the properties of the top seabed strata, down to $\sim 25 \mathrm{~m}$ below the water-seabed interface.

The physical properties of the seabed are very difficult to measure directly from samples because, if the seabed is made of sediments, samples are very soft, highly saturated in water, and are not recoverable by usual coring techniques. Using laboratory and in situ measurements Hamilton (1976) proposed a set of regression laws for the shear velocity in sediments composed of sand or silt-clay where, in the first $25 \mathrm{~m}$ of sediments, $S$-wave velocities (Vs) are predicted to range from 128 to $300 \mathrm{~m} / \mathrm{s}$. For compressional waves, Hamilton (1976) reports estimates for fine and coarse sands where the $P$-wave velocity ( $\mathrm{Vp}$ ) for the first $20 \mathrm{~m}$ range from 1700 to $1900 \mathrm{~m} / \mathrm{s}$. Taken together, these results point to a very high $\mathrm{Vp} / \mathrm{Vs}$ velocity ratio, exceeding ten, for the shallowest sediments in the ocean floor. In comparison, in terrestrial sediments and hard rock the $\mathrm{Vp} / \mathrm{Vs}$ velocity ratio rarely exceeds a value of 2.0 (e.g., Aki and Richards, 1980) and water saturated sediments typically have a ratio of 3.0 at $1000 \mathrm{~m}$ depth below the seafloor (Hamilton, 1976).

Sediment density must also be considered. Measurements published by Nafe and Drake (1963) for marine sediments show values that range from 1.3 to $2.0 \mathrm{~g} / \mathrm{cm}^{3}$ for $P$-wave velocities between 1500 and $2000 \mathrm{~m} / \mathrm{s}$.

When an acoustic plane wave impinges the ocean floor from above, the amplitudes of the reflected $P_{-}$, transmitted $P$-, and transmitted $S$-waves are characterized by three reflectivity coefficients. These can be obtained by solving the Zoeppritz equations for the elastic parameters that are typical for soft sediments found at the seafloor. When the reflectivity coefficients for this typical model are examined, it can be concluded that all of them (reflection and transmission), have an imaginary part after some critical incident angle. The value of this critical angle depends on the $P$-wave velocity of the top seabed strata but not on the $S$-wave properties and is obtained by

$$
\text { for } \alpha_{2}>\alpha_{1}, \quad \sin i_{c}=\frac{\alpha_{1}}{\alpha_{2}} .
$$

Beyond this critical angle the emergence angle in the seabed also becomes a complex number that cannot be estimated from seismic observations. Thus, the single station method described here when applied to fin whale calls is limited to incidence angles smaller than $i_{c}$. This also means that for a given source height, $h_{w}$, above the sea floor and assuming a straight line propagation in the water layer, the method will be limited to horizontal ranges smaller than the critical range $R_{c}$ given by

$$
R_{c}=h_{w} \tan i_{c} .
$$

For example, in an environment where $\alpha_{1}$, the $P$-wave velocity in the water column, is $1500 \mathrm{~m} / \mathrm{s}$ and, $\alpha_{2}$, the $P$-wave velocity at the seabed is $1800 \mathrm{~m} / \mathrm{s}$, then the critical angle is $56.4^{\circ}$.

\section{Identifying signals within the critical range}

The theoretical amplitude of horizontal and vertical ground motion recorded by the seismometer can be easily computed from the reflectivity coefficients and from the horizontal and vertical slowness for each wave (Aki and Richards, 1980). According to plane wave theory, the vertical amplitude decreases sharply close to the critical angle while the horizontal amplitude is null for a vertical incidence and increases constantly up to the critical angle.

After the critical incidence angle, since the reflectivity coefficients are complex numbers, the three seismic channels will be differently deformed in shape and will also display different time shifts. Therefore, after the critical incidence angle, the correlation between horizontal and vertical recordings is expected to be strongly degraded. Consequently, the coherency factor, $C_{O}$, should also show a very strong decrease. Therefore, $C_{O}$ may be a potentially useful classification parameter, and its utility is investigated in later sections. 
The loss of correlation and coherency for post-critical incidence angles between the vertical and horizontal channels in the seismometer is also observed between the vertical and hydrophone channel (Fig. 2). This observation suggests three additional parameters that could be used to classify fin whale calls as being inside or outside the critical range: (i) $\mathrm{CZH}$ - the maximum normalized cross-correlation observed between the hydrophone and vertical channels; (ii) $\mathrm{iZH}$ - the time lag (in samples) for which the $\mathrm{CZH}$ above is observed; (iii) $\mathrm{CZHO}$ - the normalized cross-correlation observed between the hydrophone and vertical channels at zero time lag.

Another classification parameter that could be used is the $\mathrm{S} / \mathrm{N}$. For a constant noise background, this would be analogous to an amplitude threshold. In addition, its use would avoid the use of location estimates in the presence of large levels of noise in the seismometer. Ocean bottom seismic recordings, including the frequency band where fin whale calls are recorded, are mostly affected by oceanic swell, wind waves, bottom currents, and passing ships (e.g., Webb, 1998). The ocean bottom currents are mostly tide controlled and the noise conditions due to them are site dependent. The currents interact with the OBS parts inducing Von Karman vortices and resonance of the instrument with the seafloor (e.g., Kasahara et al., 1980; Corela, 2014) at frequencies above $2 \mathrm{~Hz}$. The hydrophone records are unaffected by this current induced noise but the OBS channels could be affected, which could reduce the accuracy of other estimated selection parameters, highlighting the utility of including the $\mathrm{S} / \mathrm{N}$ as an additional classification parameter.

In all implementations of this single station method, signal amplitudes are measured by calculating the root-meansquare (rms) of the detected pulse. The noise level is also computed as the rms of the signal in a time window of the same length as the master waveform that precedes a given detected pulse. Noise and signal amplitudes for all four channels are stored during the detection process so that they can also be used for classification.
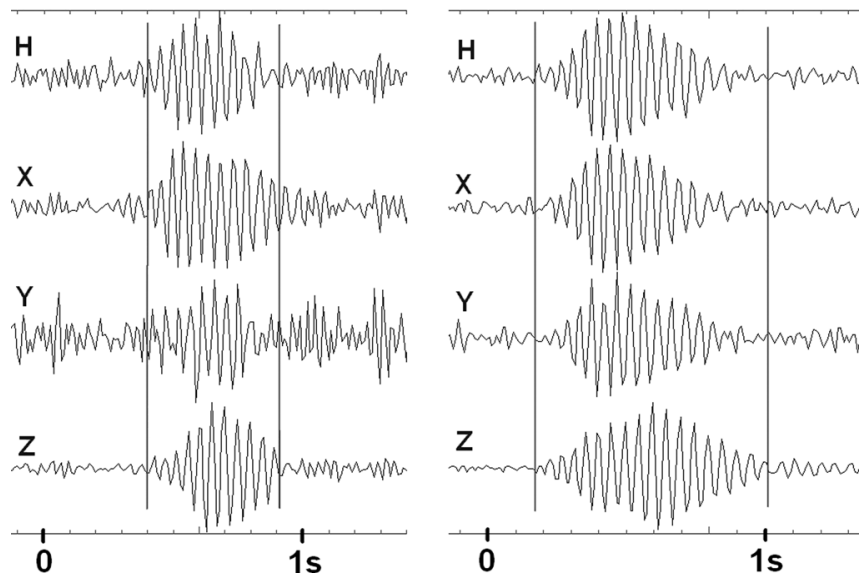

FIG. 2. Fin whale calls recorded by one seismometer showing wellcorrelated hydrophone $(H)$ and vertical signals $(Z)$ on the right panel. The left panel shows one whale call where a clear delay and difference in shape (phase) can be seen between the horizontal ( $X$ and $Y$ ), the hydrophone, and the vertical channels.

\section{Identifying multipathed signals}

Multipath arrivals must also be considered in the classification process. Despite the use of a protection time buffer, multipath arrivals may be accepted by the detection algorithm. To investigate the potential inclusion of multipath arrivals in the detection and classification processes, synthetic seismograms were computed. The seismograms simulated recordings by a seismometer at the sea floor below a water layer $4000 \mathrm{~m}$ thick using the seismic reflectivity method as modified by Wang (1999). For the first seabed layer, typical values for the elastic parameters were used: $\alpha_{1}$, the $P$-wave velocity in the water column, was $1500 \mathrm{~m} / \mathrm{s}, \alpha_{2}$, the $P$-wave velocity in the shallowest seabed layer, was $1800 \mathrm{~m} / \mathrm{s}, \rho_{1}$, the water density, was $1.0 \mathrm{~g} / \mathrm{cm}^{3}, \beta_{2}$, the $S$-wave velocity in the seabed, was $200 \mathrm{~m} / \mathrm{s}$, and $\rho_{2}$, the seabed density, was $1.5 \mathrm{~g} / \mathrm{cm}^{3}$. Some layering in the seabed down to $6 \mathrm{~km}$ depth was also included to replicate more realistic conditions at the seafloor. The synthetic seismograms for the vertical velocity, at $2 \mathrm{~km}$ spacing, are shown in Fig. 3. The travel time has a linear move-out correction with a velocity of $2 \mathrm{~km} / \mathrm{s}$ to facilitate the visualization of all significant waves.

Under this model, direct path arrivals dominate the recording up to a range offset of $18 \mathrm{~km}$ (Fig. 3). Beyond this distance the seismogram is dominated by the first multiple and the second multiple dominates beyond a range of $40 \mathrm{~km}$. However, true amplitudes of multiples are considerably attenuated beyond $10 \mathrm{~km}$, by over $-20 \mathrm{~dB}$ (this is not shown in Fig. 3 due to the relative amplitude scaling applied). These results suggest that: (1) at ranges smaller than the critical range, multipaths will have a small amplitude and so they can be removed by the detection threshold or by another amplitude-based classification parameter, such as $\mathrm{S} / \mathrm{N}$ and (2) at larger range offsets where first and second multiples

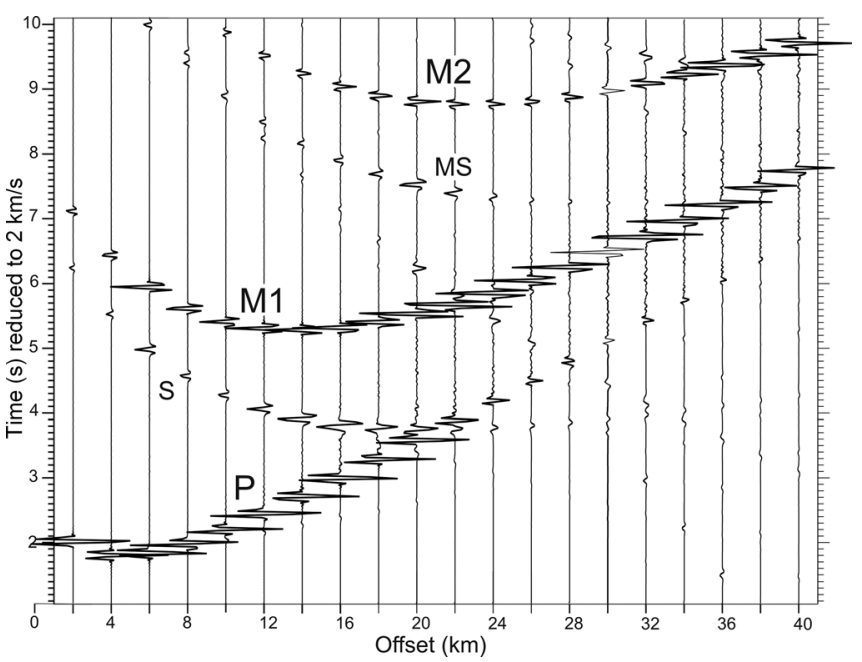

FIG. 3. Synthetic seismograms computed for the vertical component as they would be recorded by a seismometer (OBS) placed at the sea-floor below $4000 \mathrm{~m}$ of water. The travel time has a linear move-out correction applied with a velocity of $2 \mathrm{~km} / \mathrm{s}$. Amplitudes are scaled to the maximum of each trace. Hyperbolae are reflected waves while linear phases are refracted waves in the sediments. $P$-Direct path from the sound source at the surface to the OBS. M1-First multiple of $P$ after a reflection at the sea bottom and surface. M2 - Second multiple of $P$, two reflections at the sea bottom and surface. $S$ - Reflection on one sedimentary layer. MS - The first multiple of S. 
dominate, the range is so large that the reflections at the seafloor occur at angles larger than the critical angle and so the detections will be removed because of the change in shape and differences in delay between the four component channels.

The success of the proposed methodologies in the removal of super-critical incidence angles and multipath arrivals from the detected pulses can only be evaluated by the analysis of a control dataset where the true location of the acoustic sources is known. This will be examined in Sec. III.

\section{Water column properties}

Once the incidence angle of the acoustic wave on the sea floor is estimated, simple trigonometry can be used to compute the horizontal range of the sound source, assuming that the water layer is homogeneous (as in Harris et al., 2013). However, since the ocean is stratified, ray paths in the water from a near-surface source will curve considerably on their way down (concave down). When the seismic sensors are placed in deep water, this effect is expected to be large. However, the classification process should discard whale calls at range offsets larger than the critical range and for the small ranges considered by the method, the effect of ray bending by propagation in the water column is likely to be small. This was demonstrated using data from one of the test sites at the Gulf of Cadiz. In the validation analyses, the stratification of the water layer was taken into consideration.

\section{TESTING THE SSM WITH SOURCES OF KNOWN LOCATION}

\section{A. Introduction to the control datasets}

Two datasets were used to assess the method-one involved airgun signals of known location (used as a proxy for fin whale calls) and the other dataset used fin whale calls that had been localized using standard time difference of arrival methods.

\section{The active seismic survey in the Gulf of Cadiz revisited}

As part of the NEAREST project, 24 OBSs were deployed in the Gulf of Cadiz where they recorded up to 11 months of continuous four-channel data, from August 2007 to July 2008. A description of the deployment, data recorded, and instrumental characteristics were given in Harris et al. (2013) and will not be repeated here.

In September 2007, the ship R/V Atalante passed over OBS18 (4605 m water depth) and OBS19 (4287 m water depth) while producing airgun shots (Somoza et al., 2007). In Harris et al. (2013) this dataset was used to test and validate the method in its simplest form. Here, this analysis is extended with a more rigorous classification process and a more accurate ranging process, applying knowledge of water stratification and a model for the elastic parameters in the top strata of the seabed.

However, it is important to note that an airgun source used for active seismic surveying is not a perfect model for fin whale calls. While the frequencies of fin whale calls are well represented in the airgun shots, there are several key differences between the two signal types. Airgun shots have a much broader frequency spectrum and have higher energy content than the fin whale calls, affecting S/N and amplitude considerations. Furthermore, the shape of an airgun shot is different from a fin whale call. For example, while it is easy to identify on a seismogram the beginning of an airgun shot, it is not possible to do the same for fin whale calls. Finally, while the radiation pattern of a fin whale call is expected to be omnidirectional (Payne and Webb, 1971), the artificial sources used in seismic surveys are usually made of arrays of airguns tuned in such a way to focus the energy radiation vertically downwards. Therefore, it is expected that amplitudes from tuned airgun arrays will decay faster with range than fin whale calls. This is why it was important to also validate the method using a control dataset of fin whale calls.

\section{The Lucky Strike dataset}

The MOMAR ("Monitoring the Mid-Atlantic Ridge") project was initiated by the international InterRidge Programme, to study active mid-ocean ridge processes along a slow-spreading ridge section. Multidisciplinary studies have been conducted to follow up the long-term evolution of hydrothermal environments at the Mid-Atlantic Ridge near the Azores $\left(35^{\circ} \mathrm{N}\right.$ to $\left.40^{\circ} \mathrm{N}\right)$, in the Lucky Strike area. As part of the MoMAR monitoring effort, the Lucky Strike area has been continuously occupied by seismic sensors. The OBSs are periodically deployed and recovered at least once every year. The area has also been investigated by active seismic methods and the results regarding the local seismicity, geology, and crustal structure have been regularly published (e.g., Arnulf et al., 2011; Crawford et al., 2013). Besides active and passive seismic data the MOMAR sensors also recorded fin whale calls (Chauhan et al., 2009).

The data analyzed in this work belong to the BBMOMAR-2, Broad Band experiment for Monitoring of the Mid-Atlantic Ridge, 2008 cruise that recovered five OBSs in August 2008, which were deployed in July 2007 by the BBMOMAR cruise at $\sim 2000 \mathrm{~m}$ water depth. The sensors were deployed close enough to allow for the location of fin whale vocalizations using conventional seismic triangulation methods (e.g., Ottemöller et al., 2011). The dataset made available by IPGP, Institut de Physique du Globe de Paris (Crawford, 2013) comprises a set of station coordinates and waveforms with time stamps already corrected for the measured time drift after a one-year deployment. All instruments recorded at $62.5 \mathrm{~Hz}$ sampling rate to ensure 1-year continuous operation. Frequencies up to $28 \mathrm{~Hz}$ could be analyzed, which was sufficient to investigate the $20 \mathrm{~Hz}$ fin whale call.

\section{B. Methods}

\section{Signal detection}

In the Gulf of Cadiz dataset, seismic line MF12 passed straight above OBS19 and the method was tested using data from this sensor using airgun shots that originated inside a $16 \mathrm{~km}$ radius from OBS19. The analysis of the airgun shots was restricted to the time interval where direct paths in the 
water could be clearly identified. The airgun data were processed using the modified normalized cross-correlation formula shown above with $M=0.5$ [Eq. (2)] using recordings from the vertical channel. The detection threshold (Cmax) was set to 0.3 . The master waveform used for the detection process was one of the loudest airgun shots recorded ( $430 \mathrm{~ms}$ duration).

It was of interest to estimate the critical range for the Gulf of Cadiz recordings. However, the $P$-wave velocity in the shallowest sediments, $\alpha_{2}$, was not known. Therefore, assuming an average sound speed of $1.502 \mathrm{~km} / \mathrm{s}$ in the water column, varying $P$-wave velocities in the sediments were assumed, resulting in several candidate critical rang estimates: $6.49 \mathrm{~km}\left(\alpha_{2}=1.8 \mathrm{~km} / \mathrm{s}\right), 5.53 \mathrm{~km}\left(\alpha_{2}=1.9 \mathrm{~km} / \mathrm{s}\right)$, and $4.88 \mathrm{~km}\left(\alpha_{2}=2.0 \mathrm{~km} / \mathrm{s}\right)$. These predictions were compared to the detection results to see which seemed the most plausible.

The receiver operating characteristic (ROC curve) of the detector as a function of the cross-correlation threshold was also calculated. The success interval was restricted to an approximate critical range $(5 \mathrm{~km})$. True positive rate was calculated as the number of direct path signals originating within the critical range as a proportion of the total number of detections. In this analysis the false positive rate (FPR) was calculated as the number of false detections within the critical range as a proportion of the total number of false positives and true negatives. Any call originating from outside the critical range was considered to be a false positive if detected, and a true negative if undetected.

Multipaths were considered to be true negatives/false positives in the classification process, regardless of the range at which they were produced. The true positive rate (TPR) as a function of range was also assessed for different values of Cmax [using the detection algorithm in Eq. (2) where $M=0.5]$, initially without any classification applied. The TPR was calculated as the number of true positive detections performed over the total number of direct path shots inside a bin $1 \mathrm{~km}$ wide, allowing for $500 \mathrm{~m}$ overlap between bins for the Gulf of Cadiz dataset and 250 m overlap for the Lucky Strike dataset.

In the Lucky Strike dataset, recordings of fin whales at multiple stations were used to generate the control dataset against which the method was tested. Visual inspection of data showed that one of the strongest fin whale $20-\mathrm{Hz}$ call sequences was recorded on November 30, 2007, between 08:00 and 10:00, for a 2-h period. The highest amplitude call from one OBS (LSo7) was chosen as the master waveform in the matched filter analysis. The choice was made due to the simplicity of the waveform and the absence of multipath arrivals. Call detection was performed using a matched filter as explained in Sec. II A, using $M=0.5$. A low detection correlation threshold $(0.3)$ was used to ensure that most of the whale calls were detected. All detections on all five instruments were examined and corrected. OBS LSo7 provided the best quality signals and it was selected as the main call sequence to be processed using the single station method.

Next, all five individual call sequences were associated so that every single call could be identified in as many recordings as possible (with a maximum of five occurrences). This process was done by visual inspection, profiting from the fact that fin whales travel slowly while vocalizing (e.g., 4-7 km/h as reported in Rebull et al., 2006) and produce regular sequences of sounds interrupted by small gaps. The arrival times were manually re-picked at the onset of each pulse. Visual comparison of signals side-by-side facilitated the choice of a homogeneous picking point between different stations. This procedure generated a total of 427 events, with most calls being recorded by four or five instruments.

By treating the calls as earthquakes located at or close to the surface, the $2 \mathrm{D}$ call locations were obtained using the SEISAN application (Ottemöller et al., 2011), which performs seismological analyses. The velocity model in the water layer was derived from the conductivity, temperature, and depth (CTD) profiles recorded by the BBMOMAR cruise (Crawford et al., 2008). After a first location it was noted that some events diverted from the expected smooth path of a fin whale, and so events were examined to obtain an improved continuous path that represented the whale track. It was found that the conventional use of three decimal places to represent event locations inside SEISAN was not adequate for the analysis of these closely located sources, which occurred within a radius of $\sim 6 \mathrm{~km}$. Therefore, the final location was obtained with a modified high-resolution code of the SEISAN location routine. Only identified primary arrivals were used in the localization routine. The estimated locations were further improved by taking the running average coordinates of the neighboring four events for each location.

\section{Signal classification}

Both signal types (airgun shots and fin whale calls) were classified using the classification parameters presented above. Varying values for $C_{O}$ and iZH were tested. Preliminary tests showed that the $\mathrm{CZH}, \mathrm{CZH} 0$, and $\mathrm{S} / \mathrm{N}$ parameters were not as useful as $C_{O}$ and $\mathrm{iZH}$ and so no results for these parameters will be presented. The TPR was assessed as a function of range for various classification procedures. In order to assess whether the classification parameters were successful at reducing the number of false positives, which could include direct pulses from outside the critical range, the FPR was also estimated as a function of range. The FPR within each assessed distance was estimated by dividing the number of falsely classified signals (signals that were not direct paths inside the critical range) by the total number of false positives and true negatives across all ranges.

\section{Water column properties}

In the two areas where the method was tested, the Gulf of Cadiz is the one where the seismic sensors were deployed at deeper depths, from 2000 to $5000 \mathrm{~m}$. Ocean stratification was derived from CTD data that was acquired during 2007 as part of the NEAREST EC project (Lobue, 2012). The original sound speed profile was acquired on the August 24, 2007 at 19:53:41 (UTC) at a location close to OBS25 (see Harris et al., 2013 for a map of the OBS locations). The raw data were downsampled to 1 and $10 \mathrm{~m}$ intervals and linearly extrapolated from 3167 to $5100 \mathrm{~m}$. The resulting sound speed profile displayed a warm and salty water layer between 800 and $1600 \mathrm{~m}$ that represents the Mediterranean 


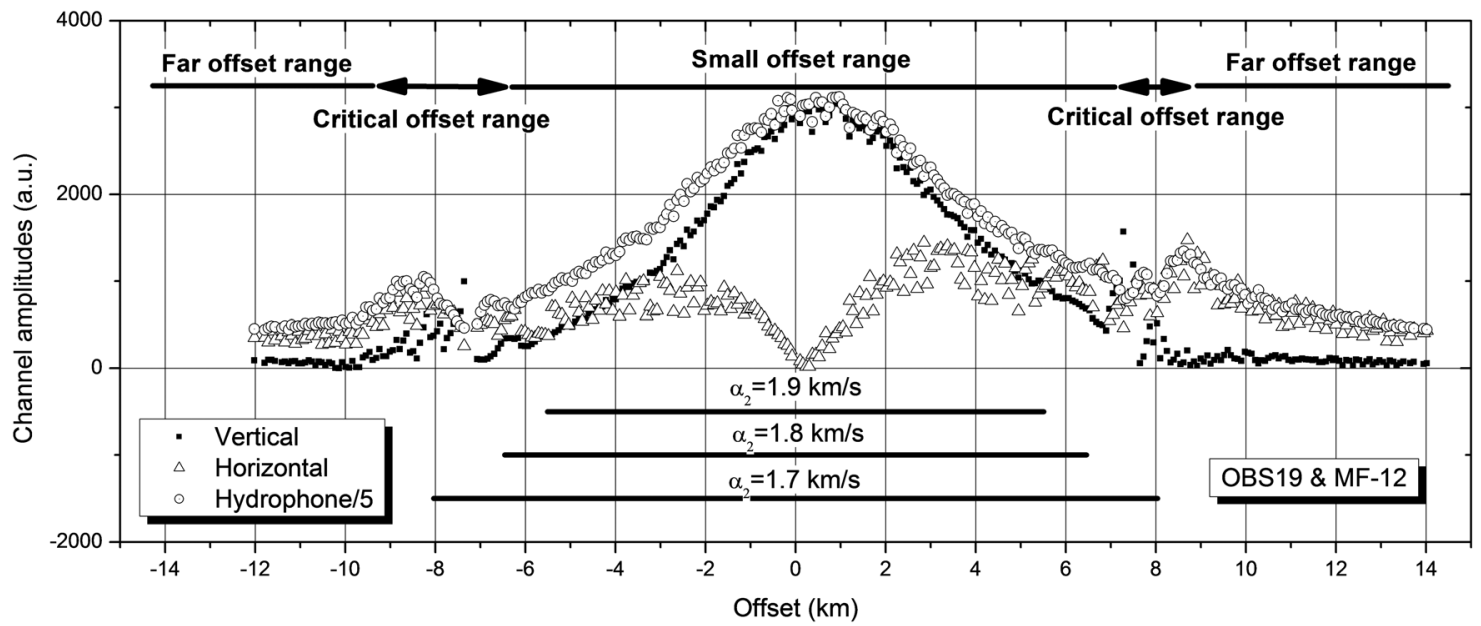

FIG. 4. Variation of airgun shot amplitudes with range offset (negative to the west). The horizontal channels are merged into a single horizontal measure. The hydrophone was scaled (divided by five) so that it could be compared with the vertical channel. Three sub-critical ranges are computed for three different $P$-wave velocities in the sediments. Three range domains can be identified with different amplitude behaviors: the small offset range, the critical offset range, and the far offset range.

Outflow Water. This layer is likely to affect the sound propagation in the Gulf of Cadiz and it is shown to be present in the whole deep-water area where the NEAREST OBSs were deployed (e.g., Ambar et al., 2008). For this reason, this sound speed profile was considered a good approximation for the average conditions in the studied area.

Ray tracing was performed on the horizontally stratified water layer integrating the propagation equations by a simple Euler rule from the seafloor to the ocean surface.

\section{Results}

\section{Signal detection}

For this analysis, signals generated by the active shooting up to an offset of $16 \mathrm{~km}$ were considered. A total of 427 airgun shots were fired within $16 \mathrm{~km}$ and a large number of multipaths were generated $(n=2090)$. In the OBS records, 346 shots were clearly identified as direct paths, 133 of which were generated inside a $5 \mathrm{~km}$ radius (representative of the critical range). The direct path arrivals of 81 shots, typically produced at larger offsets, could not be identified in the OBS records at all. However, some of the detected multipath arrivals may have originated from these shots.

A comparison of the amplitudes for the horizontal, vertical, and hydrophone channels as a function of the range offset between the airgun shots and OBS19 is given in Fig. 4. The comparison of the amplitudes in this way leads to several useful observations: (i) three domains as a function of range offset can be clearly identified: the small offset range, the critical distance range, and the far-offset range; (ii) the amplitude of the vertical $(Z)$ channel decays as expected, almost reaching zero at the critical distance and after that, oscillating close to zero; (iii) the amplitude on the hydrophone $(H)$ channel also decays but never reaches a value close to zero; (iv) the decaying of $H$ amplitudes in the small offset range looks similar to what is expected for a free instrument with amplitude attenuating with geometrical dispersion but this behavior changes drastically at critical distances and in the far-offset ranges; (v) all amplitudes show strong oscillations at the critical range; (vi) the amplitude of the horizontal ground movement is zero at zero ranges and increases steadily thereafter. After reaching a maximum, it remains high up to the far-offset range where it decays continuously. The maximum amplitude is seen just after the critical distance as would be expected from the solution of the Zoeppritz equations; (vii) the amplitude variations are not exactly centered at $0 \mathrm{~km}$ range offset reflecting $\sim 50 \mathrm{~m}$ uncertainty in sensor position after being relocated using the direct wave arrivals.

The high variability of amplitudes after the critical range supports the theory from Sec. II and empirically demonstrates that the method is reliable only in the small-offset range. Examining the candidate critical distances displayed, it appears that the most plausible value for the $P$-velocity in the sediments is $1.8 \mathrm{~km} / \mathrm{s}$ or very slightly slower.

The ROC curve showed that a $0 \%$ false positive rate was attained for a detection threshold of 0.8 or higher (Fig. 5). However, a considerable number $(30 \%)$ of true shots were

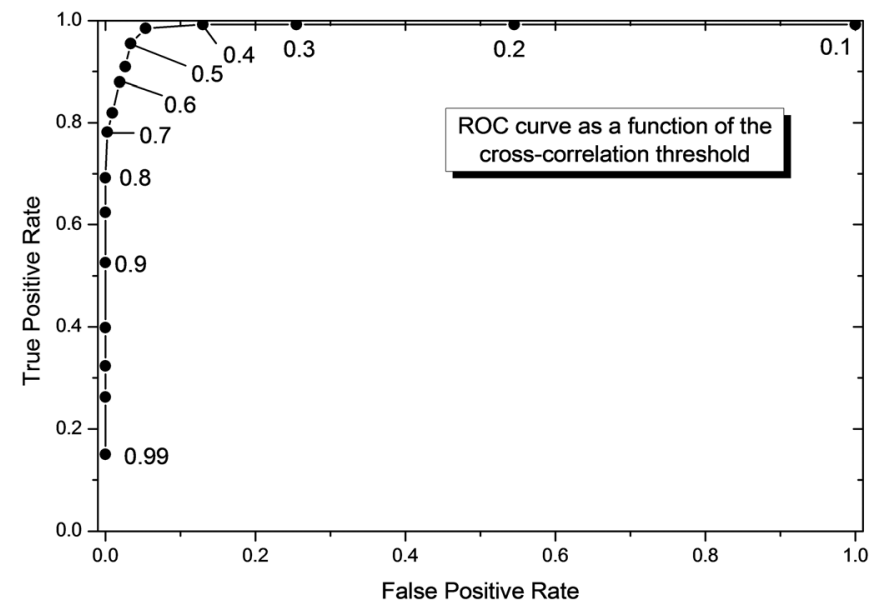

FIG. 5. ROC curve for the SSM detector as a function of the crosscorrelation threshold. Data tested includes the time interval between $12 \mathrm{~km}$ (to the west) and $14 \mathrm{~km}$ to the east. A successful range estimate could only occur inside the critical range $(5 \mathrm{~km})$. FPR is calculated as a proportion of false positives and true negatives. 
missed at that threshold level. Therefore, it may be preferable to use a conservative value for detection (e.g., 0.4) and design a classification scheme that preserves the true detections and filters out most (or all) of the false positives that originate both from inside and outside the critical range.

In the Lucky Strike dataset, OBS LSo7 generated 427 good quality calls and all the other sensors generated more than 390 whale calls. The number of fin whale call detection times available allowed the computation of $416 \operatorname{good} 2 \mathrm{D}$ locations (out of 427 possible locations) with an average rms time error of $0.045 \mathrm{~s}$. The resulting smoothed track ran roughly from north to south until the triangulation failed to

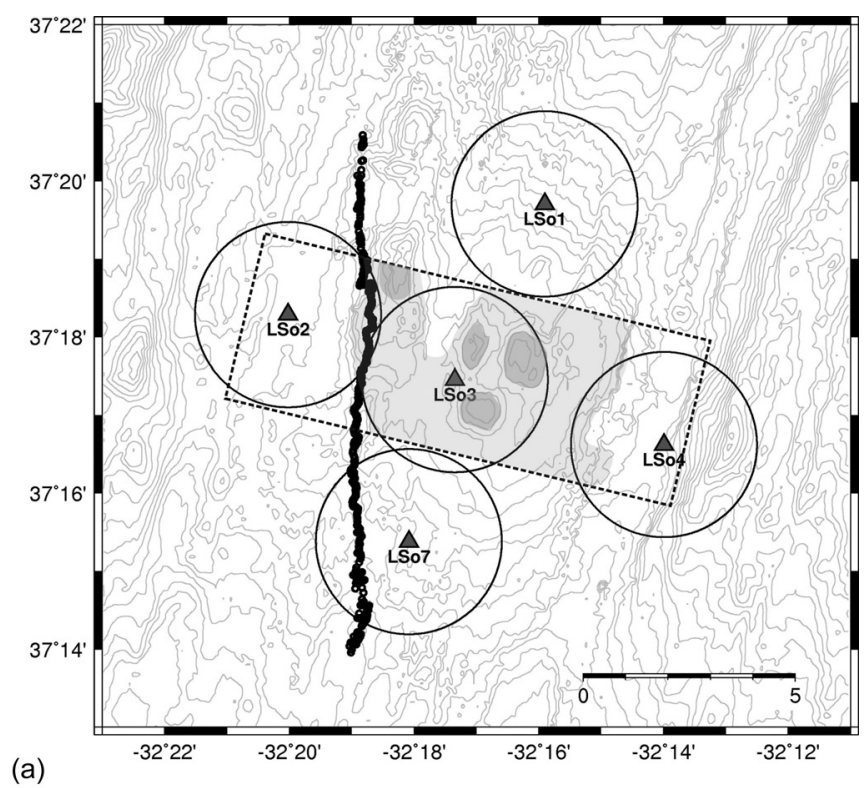

(a)

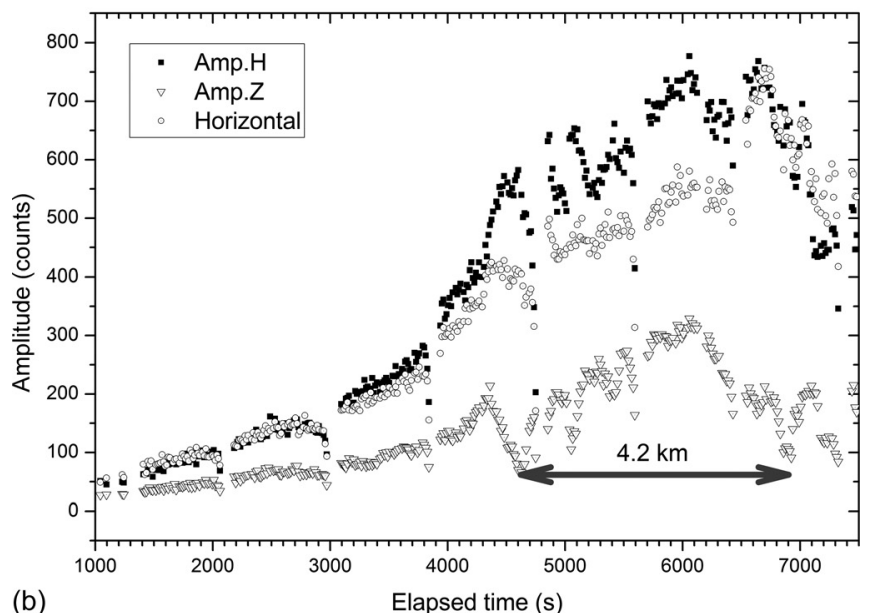

FIG. 6. (a) Location of the five sensors deployed in the Lucky Strike area (Azores). The filled black dots show the smooth track of fin whale calls identified on November 30, 2007. The circles surrounding each station mark the critical range beyond which the SSM cannot be applied ( $P$-wave velocity in the shallow seabed assumed to be $2.1 \mathrm{~km} / \mathrm{s}$ ). The dashed rectangle outlines the area covered by 3D seismic data, targeting the central volcano, where a high-resolution tomographic model for $P$-wave velocity was derived (Arnulf et al., 2011, 2012). In these models anomalous low velocities were found at shallow levels (light shaded area) with small domains of velocities of $1.8 \mathrm{~km} / \mathrm{s}$ or lower (darker shaded areas) (Arnulf et al., 2011, 2012). (b) Amplitude variation with elapsed time for primary arrivals. Amplitudes are in counts for the hydrophone $(H)$ and the seismometer components. $Z$ is the vertical channel. The horizontal amplitude is computed from $X$ and $Y$ amplitudes. The arrow shows the critical range estimated from the $Z$ component. work due to the whale being too far from the center of the array [Fig. 6(a)].

The amplitudes of the calls at far ranges (from 1000 up to $4300 \mathrm{~s}$ after the start of the time period of interest) increased continuously and smoothly as would be expected from the approaching of the fin whale to the sensor [Fig. 6(b)]. It was also clear in this time interval that the sound level generated by the fin whale dropped suddenly during the last calls of a sequence (i.e., at 2100, 3000, and 3800 s).

In theory, the amplitude of the vertical component $(Z)$ should drop to zero at the critical range. This property enables the critical range to be identified [Fig. 7(b); from 4600 to $6900 \mathrm{~s}$ ]. Since the whale track line did not directly approach LSo7 [Fig. 7(a)], the variation of amplitudes for the $Z$ channel is not very large, but a slow increase then decrease can be seen, denoting the approaching and departing of the sound source. The hydrophone amplitudes also show a slow variation, similar to the $Z$ channel, except for elapsed times larger than $6400 \mathrm{~s}$. An increase in amplitude is observed here, coincident also with an increase in the horizontal amplitude.

\section{Signal classification}

The performance of all classification parameters presented in Sec. II B were investigated and the most promising were the polarization coherency $\left(C_{O}\right)$ and the correlation lag between $Z$ and $H$ channels (iZH). The variation of threecomponent coherency, $C_{O}$, as a function of range, for direct wave arrivals inside a radius of $16 \mathrm{~km}$ is shown in Fig. 7(a) for OBS19 and line MF12. A positive value of $C_{O}$ generally identified the short-range domain except for near vertical incidence, or near-zero range, where, due to the small amplitude of the horizontal channels, the three-component coherency was poor. It was also clear that the maximum correlation (C.Max) from the detection process is very high for the near vertical incidence where $C_{O}$ was unreliable. Therefore, coherency can be a useful classification parameter if used conditionally; that is, calls should not be rejected using $C_{O}$ if the correlation is high or if the estimated range is close to zero. This protection radius inside which $C_{O}$ classification cannot be applied can be defined as a percentage of the sensor depth.

The variation of the lag for the maximum correlation between vertical and hydrophone channels with range offset, for direct wave arrivals, is shown in Fig. 7(b) for OBS19 and line MF12. Due to the phase change at critical and supercritical incidence angles, the $\mathrm{iZH}$ parameter seemed to be a good discriminator to identify the short offset domain where reliable range estimates can be performed. However, some signals close to the critical range may be rejected and some far-offset calls may be retained.

For conservative values of the detection threshold (e.g., 0.3 ) shots produced beyond the critical range were easily detected. Undesired signals (i.e., shots outside the critical range) were only filtered out at a range close to the double of the critical range $\left(R_{c}\right)$. For larger threshold values, the detector filtered out signals at range offsets close to but smaller than $R_{c}$. This behavior is the one expected based on the 

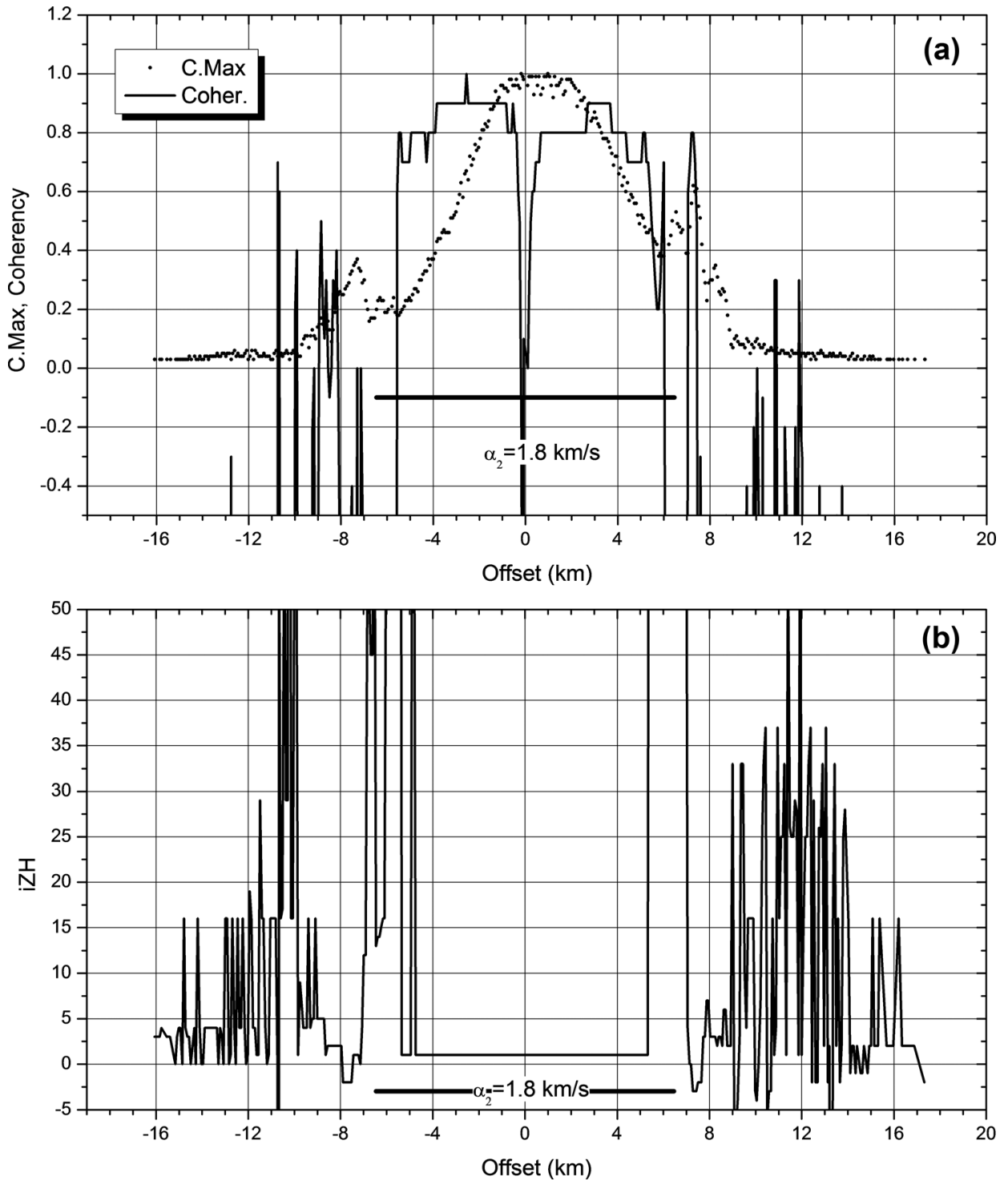

FIG. 7. (a) Variation of threecomponent coherency $\left(C_{O}\right)$ with offset for direct paths inside a $16 \mathrm{~km}$ radius surrounding OBS19. Also shown is the maximum correlation that resulted from the detection process (Cmax). (b) Variation of the lag (in samples) for the maximum correlation between vertical and hydrophone channels with offset (iZH). Direct wave arrivals selected from OBS19 recording MF12 line. variation of amplitudes of the $Z$ channel with range offset (Fig. 8). However, at range offsets larger than $R_{c}$, coinciding with an increase in the $Z$ channel amplitude, the detector was unable to filter out a number of undesirable signals. Without using any other selection criteria, the TPR at $\sim 7 \mathrm{~km}$ could be reduced by increasing the detection threshold, but with the effect of reducing the number of true classifications inside the critical range.

Several classification values were tested for the Gulf of Cadiz active seismic survey and it was found that $\mathrm{iZH} \leq 1$ was a good discriminator. The detector performance at close ranges did not alter but signals originating beyond the critical range were more successfully rejected [Fig. 8(b)]. However, a small and undesirable window for positive detections beyond the critical range remained at $\sim 7 \mathrm{~km}$ range offset.

The polarization coherency between the vertical and horizontal channels, $C_{O}$, was the other classification parameter that performed best to reject signals from outside the critical range, using a $C_{O} \geq 0.3$ as a threshold and a protection radius (where $C_{O}$ classification was not used) of $250 \mathrm{~m}$ [Fig. 8(c)].

Finally, the classification results were improved when both parameters were used together [Fig. 8(d)]. It seems that the two criteria reject different pulses and so the performance of the classifier at range offsets larger than $R_{c}$ was considerably improved.

The FPR was only calculated for the classifier that joined the iZH and $C_{O}$ classification parameters [Fig. 8(e)]. The FPR was small $(<0.003)$ for distances larger than $5 \mathrm{~km}$ and for a detection threshold of 0.4 or larger.

The results for the detection and classification of the fin whale calls at the Lucky Strike site were very similar to the results from the Gulf of Cadiz dataset [Figs. 9(a)-9(c)].

For the lowest thresholds examined $(\mathrm{Cmax}=0.5,0.55)$ calls produced from beyond the critical range $\left(R_{C}=2.0 \mathrm{~km}\right.$ for a $P$-velocity in the sediments of $2.1 \mathrm{~km} / \mathrm{s}$ ) were easily detected. For larger values of the threshold, the detector started to filter out calls at range offsets close to, but smaller than, $R_{C}$. At offsets larger than $R_{C}$, coinciding with an increase in the $Z$ channel amplitude [Fig. 6(b)], the detector was unable to filter out a number of undesirable pulses.

Values of $C_{O} \geq 0$ were found to be good classification criteria but the application of iZH was not as simple as for the airgun validation test. Most of the direct arrivals displayed a value for iZH of 2, with a different threshold close 

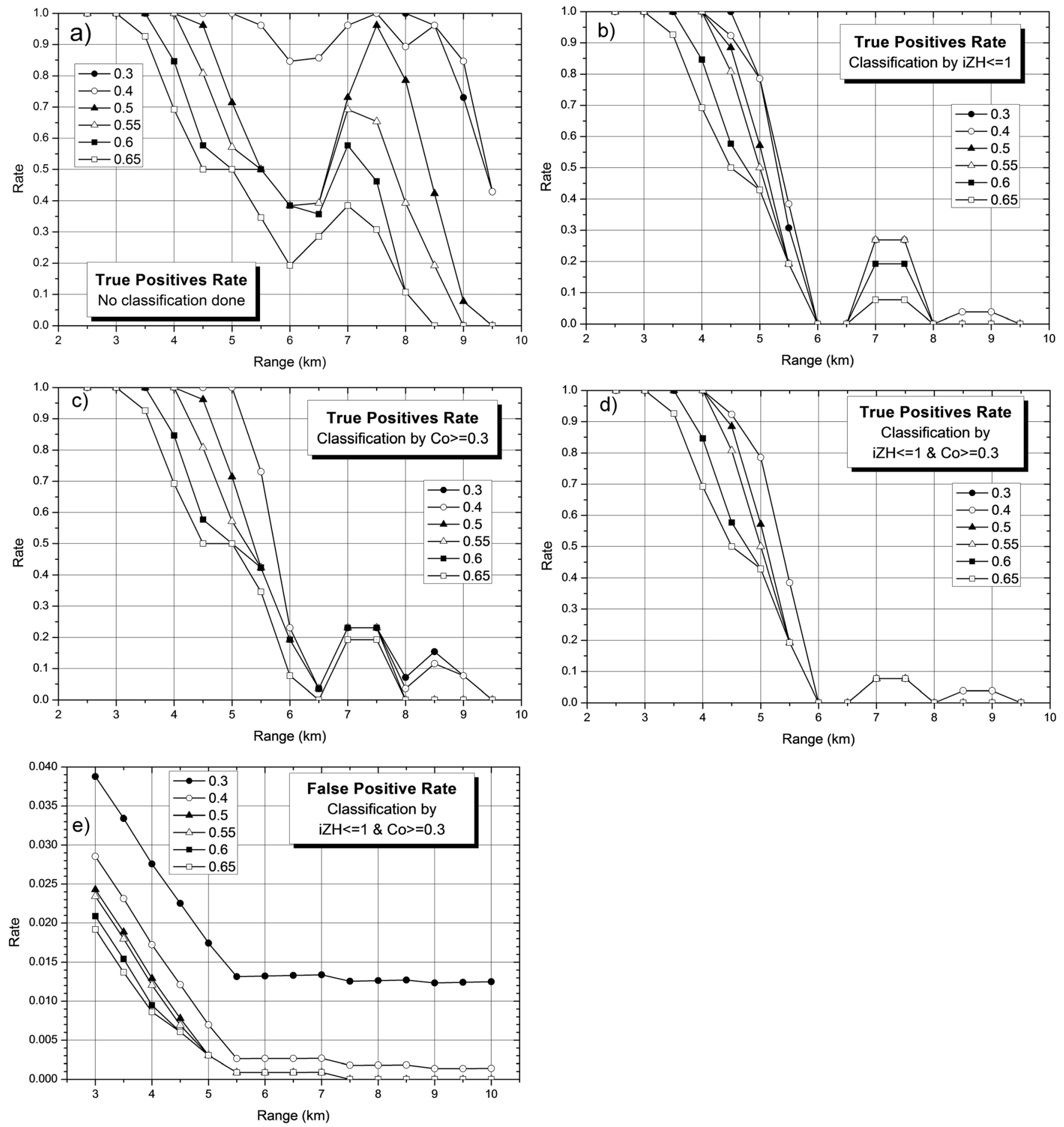

FIG. 8. TPR and FPR computed for direct paths from airgun shots, as a function of distance, for different values of the correlation threshold. Each data point represents the TPR and FPR inside a $1 \mathrm{~km}$-wide bin (with a $500 \mathrm{~m}$ overlap between bins). FPR is calculated as a proportion of false positives and true negatives. (a) TPR calculated from detections exceeding the threshold with no further classification; (b) TPR calculated from detections with classification using the delay between the $Z$ and $H$ channels (iZH); (c) TPR calculated from detections with classification using the polarization coherency $\left(C_{O}\right)$; $(\mathrm{d})$ TPR calculated from detections with classification using both iZH and $C_{O}$. (e) FPR as a function of distance, for different values of the correlation threshold. The estimated critical range is $5 \mathrm{~km}$.

to 0 for multipaths and post-critical incidence angles. The increased iZH values required to identify direct path calls may be due to a minor lag between the horizontal and vertical channels attributed to some uncorrected effect in the digitizer. The poor discrimination displayed by iZH is due to the low sampling rate for the dataset, $62.5 \mathrm{~Hz}$, implying that each sample represents a significant shift for a $\sim 20 \mathrm{~Hz}$ fin whale call. When both classification criteria were used together, the combined action of both parameters successfully rejected calls from outside the $R_{C}$ [Fig. 9(b)].

A total of 287 false triggers were identified. The FPR for the classifier that combined iZH and $C_{O}$ selection parameters is small for distances larger than $2 \mathrm{~km}$ and for a detection threshold of 0.55 or larger [Fig. 9(c)]. 

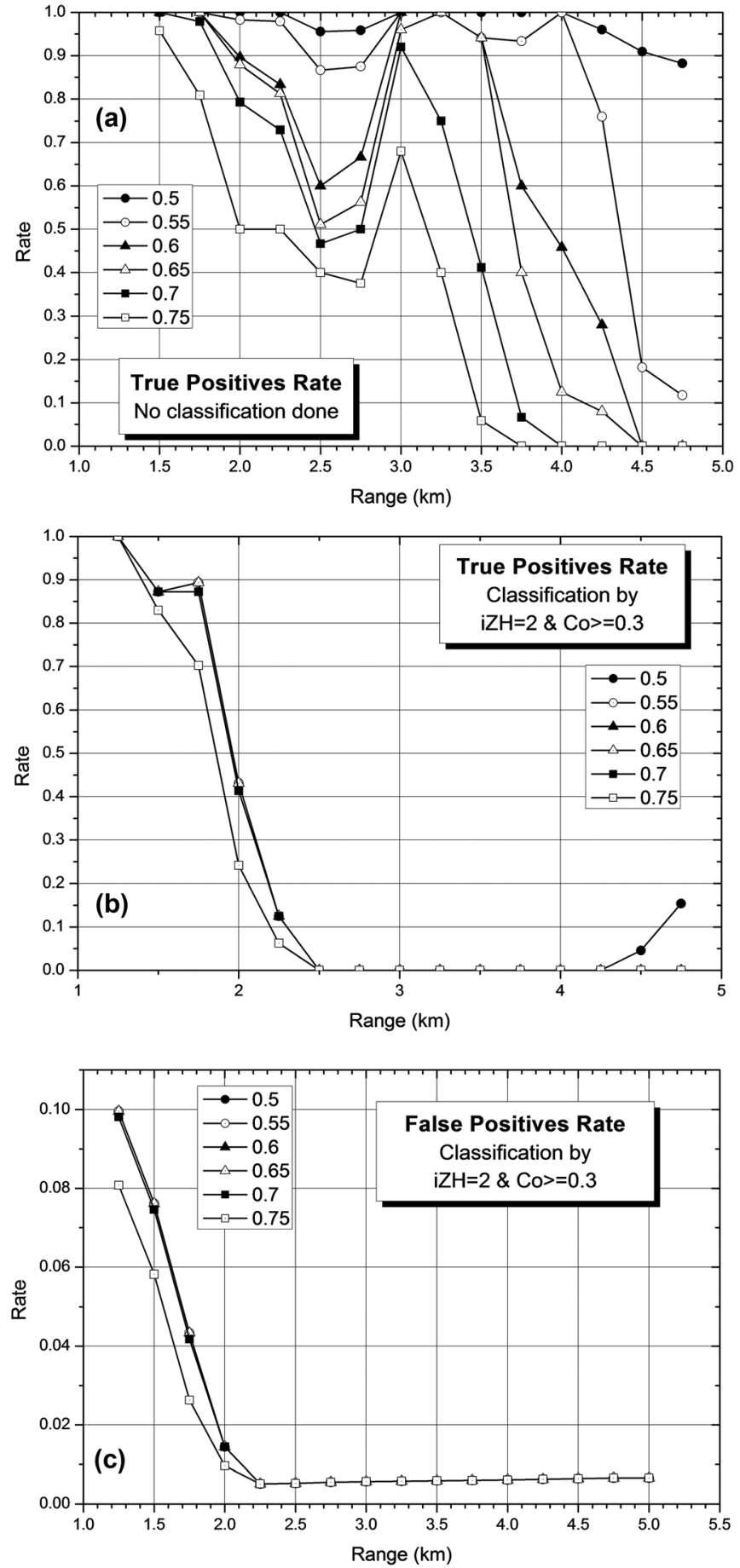

FIG. 9. TPRs and FPRs for the classification of the direct paths from known fin whale calls, as a function of distance, for different values of the correlation threshold. Each data point represents the rate computed inside a $1 \mathrm{~km}$-wide bin, with a $500 \mathrm{~m}$ overlap between bins. FPR is calculated as a proportion of false positives and true negatives. (a) TPR calculated from detections exceeding the threshold with no further classification; (b) TPR calculated from detections with classification using both the delay between the $Z$ and $H$ channels (iZH) and the polarization coherency $\left(C_{O}\right)$. (c) FPR as a function of distance, for different values of the correlation threshold. The estimated critical range is $2 \mathrm{~km}$.

\section{Water column properties}

Using the Gulf of Cadiz data, it was concluded that assuming a homogeneous sound speed in the water column resulted in estimates of horizontal range that exceeded the true range computed for a realistic sound speed profile. The relative error in horizontal range estimates increased slowly from $2 \%$ to $5 \%$ at the border of the critical range.

\section{ACCOUNTING FOR THE SEABED STRUCTURE IN THE RANGE ESTIMATES}

In Harris et al. (2013), the Gulf of Cadiz data were used for the first evaluation of the method but several simplifying assumptions were considered: (i) the $S$-wave velocity in the shallow layers of the seabed was assumed to be zero (pure acoustic propagation was applied); (ii) the water stratification and its effects on ray propagation in the ocean were neglected. Harris et al. (2013) showed the effect of (i) is likely to be small for water-saturated sediments and using ray tracing in this study showed that the effect of assumption (ii) is small.

Harris et al. (2013) made a comparison between the true and estimated horizontal ranges but did not comment on a specific pattern in the bias between true and estimated horizontal ranges - the method over-estimates true ranges at intermediate range offsets, but seems to be correct close to the critical distance (Fig. 10). The maximum difference is $\sim 1 \mathrm{~km}$ but it is only a few hundred meters on average. There is also a slight asymmetry between the two sides of the shooting. This feature can be attributed to the directivity of the source and remaining errors in the instrument coordinates after relocation. However, the intermediate range bias

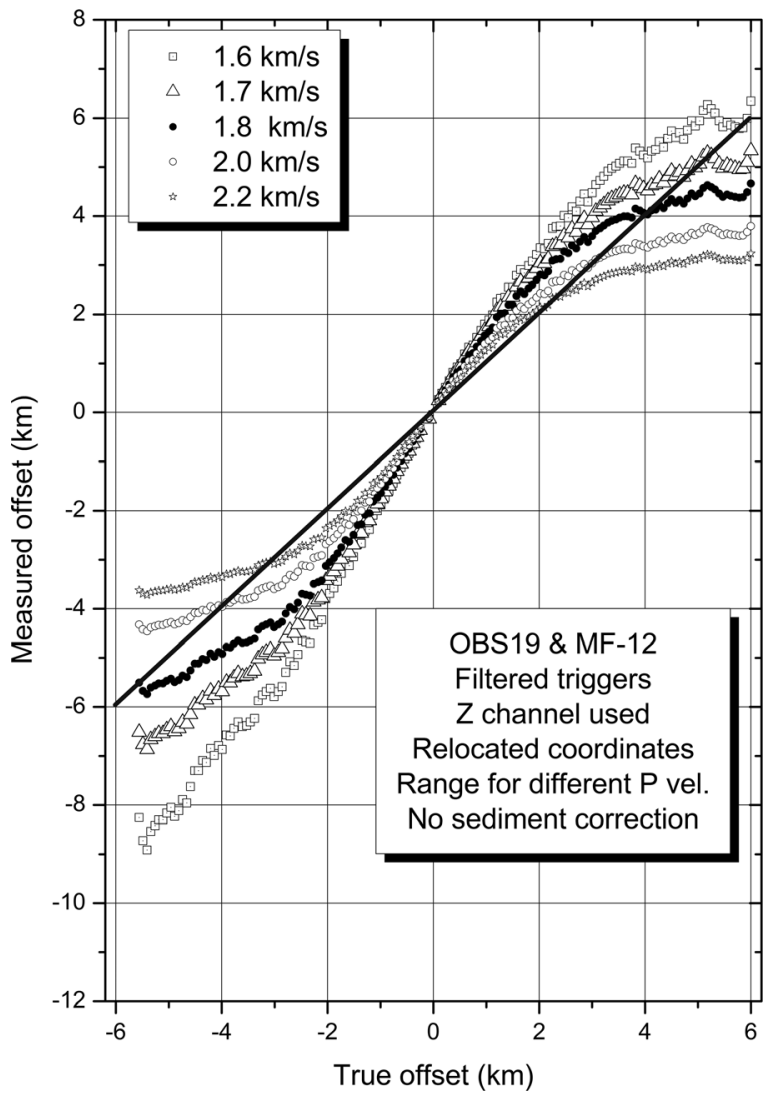

FIG. 10. Comparison of true and measured offsets for OBS19 recording MF-12 airgun shots. Offsets were computed neglecting the sediment structure and assuming straight rays in the water layer. The sound speed in the water was assumed to be $1.5 \mathrm{~km} / \mathrm{s}$. Filled circles show our preferred solution. 
was further investigated by considering the influence of the sedimentary structure on the recorded amplitudes in detail. In the following results the ray bending effect in the water layer propagation, though small, was corrected using the measures sound speed profiles.

\section{A. Seabed properties}

The simplest approach that considers the influence of the shallow seabed properties is to assume that there are no $S$-waves propagating in the sediments, as in Harris et al. (2013). Then the apparent angle measured by the seismic records is given by the Snell law. By varying the shallow $P$-wave velocity from 1.7 to $2.5 \mathrm{~km} / \mathrm{s}$ the minimum average error $(356 \mathrm{~m})$ was obtained for a $P$-wave velocity of $1.9 \mathrm{~km} / \mathrm{s}$. However, the bias in ranging remained.

To fully consider the effect of the sedimentary layer, a realistic model for both the $P$ - and $S$-wave velocities and density must be provided and seismic amplitudes must be computed by solving the appropriate Zoeppritz equation. From the analysis of Gulf of Cadiz data, the $P$-wave velocity was assumed to be close to $1.8 \mathrm{~km} / \mathrm{s}$. Investigations done in areas with soft sea-floor sediments, as expected in the Gulf of Cadiz, have shown that the first 100 to $200 \mathrm{~m}$ of sediments are very soft and nearly saturated in water. Estimates of $\mathrm{Vp} / \mathrm{Vs}$ ratios can attain 10 for this very first layer (Shipboard Scientific Party, 1972; Hamilton, 1976; Buckingham, 1998; Crawford and Singh, 2008). By making a systematic evaluation of $P$ - and non-zero $S$-wave velocities the best fit was obtained for $\mathrm{V}_{\mathrm{P}}=2.0 \mathrm{~km} / \mathrm{s}$ and $\mathrm{V}_{\mathrm{S}}=0.1 \mathrm{~km} / \mathrm{s}$ (average error $=402 \mathrm{~m}$ ). Using the above knowledge of the shallow sediments found in the deep basins of the Gulf of Cadiz and assuming that $\mathrm{V}_{\mathrm{P}}=1.8 \mathrm{~km} / \mathrm{s}$ and $\mathrm{V}_{\mathrm{S}}=0.2 \mathrm{~km} / \mathrm{s}$, the resulting average error is $720 \mathrm{~m}$, nearly twice the one that uses the simpler acoustical approach. Therefore, applying the Zoeppritz equations using realistic sediment velocities worsens the fit between known and estimated ranges and does not solve the observed bias.

Geological sampling and mapping show that the Lucky Strike site is covered by various volcanic deposits, mostly lava basalts but also breccias that evidence explosive volcanic activity (Arnulf et al., 2012 and references therein). The $P$-wave velocity structure in these areas has been derived from refraction and wide-angle modelling (Seher et al., 2010), three-dimensional seismic tomography (Arnulf et al., 2011), and full waveform inversion of downward continued seismic reflection data (Arnulf et al., 2012). Surprisingly, these data show that the shallowest layers (down to $300 \mathrm{~m}$ below sea floor) close to the central volcano have abnormally low velocities (Fig. 6). The local $P$-wave velocity can be $1.8 \mathrm{~km} / \mathrm{s}$ or lower (Arnulf et al., 2012, Fig. 6 ). These velocities, which present a sharp contrast with those found in deeper layers, have been interpreted as the result of strong fracturation and large porosity. Using a twophase model (basalt and seawater) Arnulf et al. (2011) showed that porosity estimates depend on the assumed shape and aspect ratio for the water inclusions. At $100 \mathrm{~m}$ below the sea bottom, porosity values can attain $57 \%$ for the most conservative models (ibid.), implying a low $S$-wave velocity and high $\mathrm{Vp} / \mathrm{Vs}$ ratio.

At the Lucky Strike site, the sensor investigated (LSo7, Fig. 6) is outside the area investigated by high-resolution seismics but it is in the continuation of the anomalous low $P$-wave velocity domain. Therefore, it can be concluded that, despite the very different geological environments, the physical properties of the shallowest layers in the Gulf of Cadiz and Lucky Strike areas are very similar. Therefore, as with the Gulf of Cadiz airgun analysis, a systematic search of possible models was conducted. The best model, with an average error of $135 \mathrm{~m}$, was given by $\mathrm{V}_{\mathrm{P}}=2.2 \mathrm{~km} / \mathrm{s}$ and $\mathrm{V}_{\mathrm{S}}=0$. The velocity for $P$-waves seemed reasonable, but the $S$-wave velocity did not. The comparison between true (derived by triangulation and smoothing) and estimated range offsets for this best model is shown in Fig. 11. The dashed lines define the time interval (to the right of the vertical line) and range interval (below the horizontal line) that were used in the systematic search $(\mathrm{R} \leq 1.8 \mathrm{~km})$. The fit was good but a bias was inferred-closer ranges were over-estimated while larger ranges were under-estimated.

\section{B. Amplitude of the horizontal channel}

The largest bias in the range estimates computed for the Gulf of Cadiz control dataset occurred when the horizontal amplitudes on the seismometer were largest (Fig. 4). This suggests that a better fit of the ranges can be obtained by applying a correction factor to the amplitude of the horizontal channel. The apparent incidence angle at the seabed is estimated from the horizontal and vertical seismic recordings by a procedure that is equivalent to

$$
\tan \left(i_{\text {app }}\right)=\frac{H}{V} .
$$

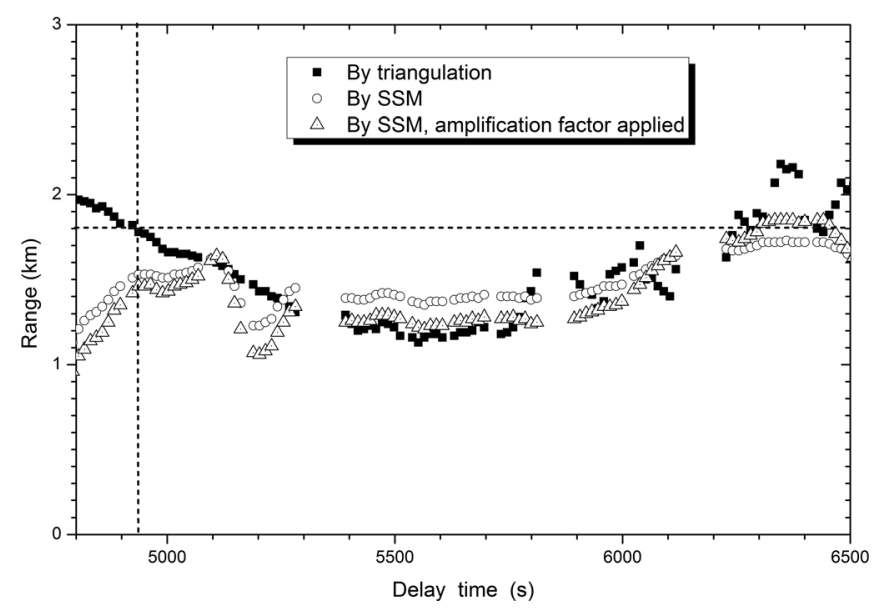

FIG. 11. Comparison of true (filled squares) and measured offsets (open symbols) for the Lucky-Strike fin whale calls. The best choice of elastic parameters in the shallowest rocks is used $\left(V_{P}=2.0 \mathrm{~km} / \mathrm{s}, V_{S}=0.3 \mathrm{~km} / \mathrm{s}\right)$. Open circles show the results without an amplification factor applied (average error is $135 \mathrm{~m}$ ). Open triangles show the results when a factor of 0.5 is applied to the horizontal amplitudes (average residual is $130 \mathrm{~m}$ ). The dashed lines define the time interval (to the right of the vertical line) and range interval (below the horizontal line) that were used in the evaluation of the SSM performance. 
Here $H$ and $V$ represent the signal amplitudes of the horizontal and vertical velocities measured by the seismometer. If a correction factor $F$ is applied to the horizontal amplitudes then a different apparent incident angle would be obtained in the seabed

$$
\tan \left(i_{\text {app }}^{*}\right)=F \frac{H}{V} \quad \text { and } \quad \tan \left(i_{\text {app }}\right)=\frac{\tan \left(i_{\text {app }}^{*}\right)}{F} .
$$

Including this amplitude factor, $F$, in the estimation of the apparent incidence angle, produced a biased estimate very similar to the one observed in the Gulf of Cadiz dataset. Different values of the amplitude factor were tested and the bias was considerably attenuated, although the error increased for large range offsets. A factor of $F=0.5$ seemed the most adequate to produce a near straight line relationship between true and estimated ranges.

To obtain the best propagation parameters, a systematic search among several possible models with varying $P$ - and $S$-wave velocities for $F=0.5$ was conducted. The model that provided the best fit between known and estimated ranges had a $P$-wave velocity of $1.7 \mathrm{~km} / \mathrm{s}$ and an $S$-wave velocity of $0.3 \mathrm{~km} / \mathrm{s}$. The final fit between estimated and true ranges for the best-fit model had an average error of $273 \mathrm{~m}$ but it was less than $50 \mathrm{~m}$ at ranges smaller than $3 \mathrm{~km}$ (Fig. 12). Larger differences still occurred at the larger range offsets and some asymmetry persisted.

The range bias in the estimates obtained for the Lucky Strike dataset is clear but less pronounced [Fig. 12(b)]. Therefore, as for the Gulf of Cadiz, a systematic search of possible models was performed, including an amplitude factor of 0.5. A slight improvement in the average residuals was obtained (135 to $130 \mathrm{~m}$ ) and the best model was much more realistic, $\mathrm{V}_{\mathrm{P}}=2.0 \mathrm{~km} / \mathrm{s}, \mathrm{V}_{\mathrm{S}}=0.3 \mathrm{~km} / \mathrm{s}$, considering the geological and geophysical characterization of the site. The bias for close ranges completely disappeared (Fig. 11).

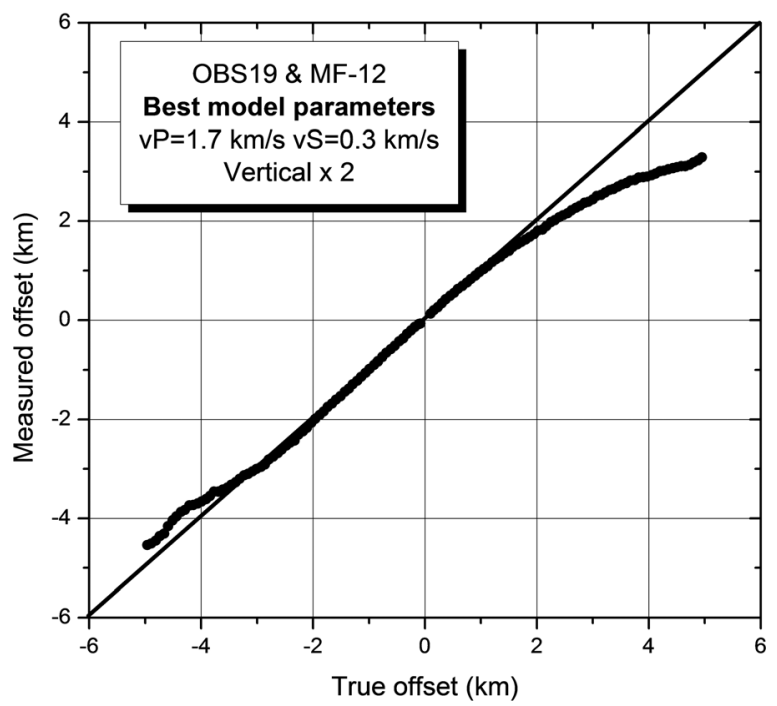

FIG. 12. Comparison of true and measured offsets for OBS19 recording MF-12 airgun shots. Here the best choice of elastic properties in the sediments derived by a systematic search was used, plus an amplification factor of two applied to the vertical channel.

\section{The amplitude factor-a possible cause}

The systematic bias in range estimates was seen at two different sites, with two different seismic signals (fin whale calls and airgun shots in the $\sim 20 \mathrm{~Hz}$ range), recorded by two different types of instruments. In both cases, a better fit between estimated and known ranges was obtained by applying an amplitude factor $(F=0.5)$ to the horizontal channels. An instrumental anomaly does not seem to be an adequate explanation, as the anomaly would have to be shared between two very different sets of instruments. Therefore, a physical explanation is required.

The use of Zoeppritz equations to derive seismic amplitudes is only valid for plane waves propagating with an infinite frequency. Fin whale vocalizations are generated by point sources and constitute bandlimited signals in frequency. To verify the results derived by the Zoeppritz equations, seismic amplitudes and apparent angles were obtained by the computation of synthetic seismograms using the seismic reflectivity method as modified by Wang (1999). A point source at $20 \mathrm{~m}$ depth was considered and a typical fin whale call was used as source wavelet. The velocity model used for the test comprised a water layer of $4000 \mathrm{~m}$ above a half space with $\mathrm{Vp}=2.0 \mathrm{~km} / \mathrm{s}$ and $\mathrm{Vs}=0.3 \mathrm{~km} / \mathrm{s}$. The differences between a very sharp transition between the two domains and a gradual transition within a thin layer $10 \mathrm{~m}$ thick were also tested. The comparison between the apparent angle obtained for different models as a function of the incident angle at the seafloor is shown in Fig. 13(a). The critical angle for this model is $49.2^{\circ}$.

The amplitudes estimated from synthetic seismograms are considerably different from those estimated by the Zoeppritz equations and vary considerably with the position of the sensor in relation to the interface between the two media. When the sensor is just above this interface then synthetic seismograms show that the amplitude ratio between horizontal and vertical signals is considerable changed, as if the horizontal amplitudes were amplified. The factor of 0.5 empirically used simply approximates this behavior, particularly at the shorter ranges (i.e., at smaller incidence angles). Comparing the average errors between the synthetic seismogram values and those estimated from the Zoeppritz equations as a function of the amplitude factor, the value of 0.5 is the one that provides the best fit for the sharp transition model and the second best when the transition model is used [Fig. 13(b)]. These results justify the choice of the amplitude factor used in the analysis of the Gulf of Cadiz and Momar site datasets.

\section{DISCUSSION}

\section{A. Method performance- Detection and classification}

The aim of the single station method proposed in Harris et al. (2013) was to derive reliable range estimates of fin whale calls from OBS recordings that can be used in distance sampling analyses. Given the properties of the interaction between the acoustic waves in the water and the shallowest sediments where the sensor is deployed, the method is limited to a critical distance that depends on the sound speed in the water and $P$-wave velocity in the sediments. Inside this 

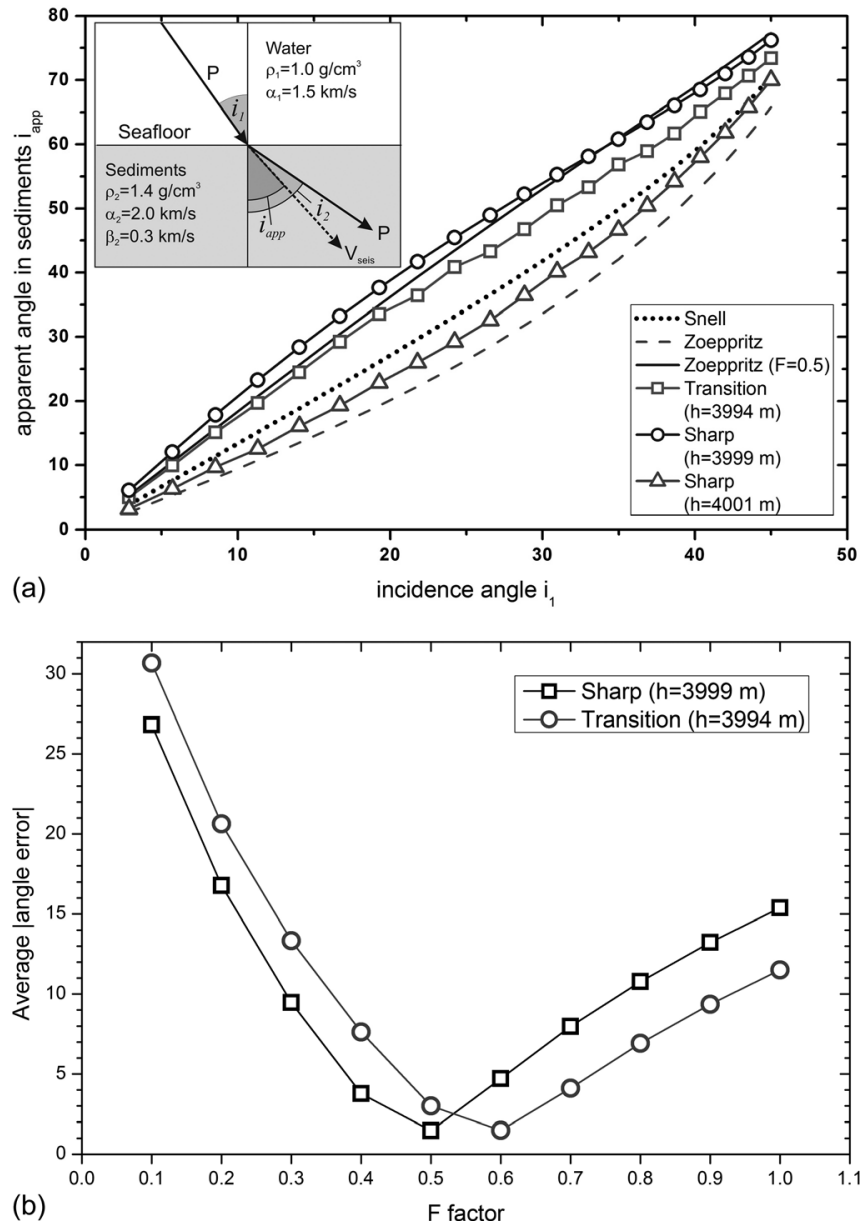

FIG. 13. (a) Apparent emergence angle in the sediments computed by different methods as function of the incidence angle at the sea-floor. The meaning of the angles and velocity model is shown in the inset. $h$ is the sensor depth. The meaning of the functions represented are as follows: Snell, computed by Snell law as if the $S$-wave velocity in the sediments was null; Zoeppritz, computed using the Zoeppritz equations; Zoeppritz $(F=0.5)$, computed using the Zoeppritz equations with horizontal amplitudes multiplied by the $F$ factor; Sharp $(h=3999 \mathrm{~m})$, computed from synthetic seismograms for a sharp transition with the sensor placed $1 \mathrm{~m}$ above the sea floor; Sharp $(h=4001 \mathrm{~m})$, computed from synthetic seismograms for a sharp transition with the sensor placed $1 \mathrm{~m}$ below the sea floor; Transition $(h=3994 \mathrm{~m})$, computed from synthetic seismograms for a gradual transition ( $10 \mathrm{~m}$ layer) with the sensor placed $6 \mathrm{~m}$ above the sea floor. (b) Average of absolute errors between the Zoeppritz model and synthetic seismogram amplitudes as a function of the amplitude factor for two of the earth models investigated, the sharp transition with the sensor $1 \mathrm{~m}$ above the sea floor and for the gradual transition.

critical range the method locates the sound sources close to the surface by measuring the amplitudes of the vertical and horizontal ground velocities and converting these to ranges by considering the water layer stratification and the elastic properties of the sediments (both $P$ - and $S$-wave seismic velocities). The azimuth of the sound source is also evaluated so that the relative 2D location of the fin whale can also be obtained. Absolute locations require the knowledge of the seismometer orientation to be inferred from active seismic surveys or passive monitoring of surface waves or body waves (e.g., Corela, 2014).

In order to be able to use the ranges generated by the method for distance sampling, there are two main criteria that must be met: (1) the measurements of range must be as accurate as possible and (2) calls produced at zero, or close to zero, horizontal distance from the instrument must be detected with certainty. These are two of the main assumptions of distance sampling and biased animal density estimates can result if these assumptions are violated (Buckland et al., 2001). Therefore, it was important to confirm that, first, the method could detect fin whale calls inside the critical range and discard all calls generated from sources outside the critical range and all multipath arrivals generated at all ranges. This would ensure that the first assumption was met. It is important to note that false positive detections are routinely dealt with in animal density estimation methods using acoustic data (Marques et al., 2013). However, in this case, false positives caused by calls originating from outside the critical range and multipath arrivals will not only bias the number of observations but also generate false range estimates that will affect the estimation of the probability of detection, a key parameter used in distance sampling (Buckland et al., 2001). Second, it was important to ensure that the algorithm used to identify direct path calls from inside the critical range was not so conservative that direct path calls produced at close horizontal distances to the instrument were rejected. This would ensure that the second assumption was met. Therefore, the detection and classification process of this single station method was thoroughly explored in this paper using two control datasets: (i) artificial airgun shots in the Gulf of Cadiz and (ii) fin whale calls recorded in the Azores (at the Lucky Strike area) located by triangulation. Using both datasets, it was verified that the detection procedure, based on a matched filter and a modified normalization of the cross-correlation, was very effective in detecting nearly $100 \%$ of the pulses that originated inside the critical range. The most promising classification parameters identified rely on the property that calls originating from outside the critical range cause a phase difference between the four recording channels (the hydrophone, vertical, and horizontal channels). This phase difference causes a lag between the hydrophone and vertical channels and degrades the polarization coherency between the three seismic channels. The classification procedure was very effective when tested on both datasets. The resulting TPR decayed from one close to the critical range (following the expected reduction in the amplitude of the vertical channel) and was small or null for range offsets larger than the critical range. The FPR considering the number of false triggers in the sequence was estimated and was shown to be small for range offsets larger than the critical range and small to nil within the critical range. Multipaths can have higher amplitude than direct paths, which may be problematic, but their amplitude only exceeds the amplitude of primary arrivals at far range offsets (approximately three times the critical range) and also close to the critical range. Therefore, multipaths could be filtered out by their amplitude or phase difference between channels.

\section{B. Method performance-Ranging}

In Harris et al. (2013) the Gulf of Cadiz active seismic survey signals were used to show the reliability of the range 
estimates generated by the method, assuming some simplifying hypotheses. Here the Gulf of Cadiz analysis was expanded by considering the effect of the water layer stratification and the elastic properties of the sediments. Since the method presented here only applies to small ranges (up to the order of the water depth), the effect of sound propagation in the water was shown to be small but can be corrected using information provided by climatological databases for the water column properties.

As regards the properties of the sediments at the seafloor, they are not known with precision at the sensor locations. For example, the shallowest sediments in the Gulf of Cadiz are thought to consist of soft pelagic sediments with a low $P$-wave velocity $\left(1.8\right.$ to $2.0 \mathrm{~km} / \mathrm{s}$ ) and a high $\mathrm{V}_{\mathrm{P}} / \mathrm{V}_{\mathrm{S}}$ ratio (Harris et al., 2013). If the $S$-wave propagation in the sediments is disregarded, then the Snell law applies. This was the simplifying assumption used in Harris et al. (2013). The analysis of the two control datasets showed that if the ideal Zoeppritz equations are applied to the recording of seismic data at the seafloor, then any physically reasonable model for the sediment properties worsens the fit between known and estimated ranges. After a systematic search of parameters, an improvement in the range fit was observed when an empirical factor of 0.5 was applied to the amplitude of the horizontal channels. Using synthetic seismograms, it was verified that the ratio between horizontal and vertical amplitudes recorded at the seafloor by the OBS approximately follows this empirical factor. The amplitude factor appears to be a simple approximation, which can be applied to account for the departure from ideal Zoeppritz equations when the equations are used to estimate the range to a whale call/airgun shot.

\section{Conclusions}

Data recorded by OBSs, comprising three ground velocity channels and one pressure channel, can be used to identify and locate fin whale vocalizations. The procedure outlined in Harris et al. (2013) and detailed in this work can only be applied to fin whale calls that are direct paths to the sensor and that are incident at the sea-bottom with an angle smaller than the critical incidence that depends on the $P$ wave velocity in the sediments. To recover the sound source on the surface the method uses the ground velocity amplitude measures by the seismometer and corrects the apparent propagation angle taking into account the elastic properties of the sediments. Finally the range and location are derived using the sound speed profile in the water layer. The method also requires that the shallowest seabed properties are estimated. In the deep ocean these are of pelagic origin and their properties can be reasonably inferred. However, in very recent oceans, like the Lucky Strike site in the Azores, high porosity and highly fractured volcanic rocks have resulted in low seismic velocities that favor the application of the method. Furthermore, the deeper the OBS, the larger the critical range, within which the method can be applied. This method is then best suited for deep-water deployments on soft sediments or low velocity rocks. Fin whale calls were detected by a matched filter with a modified crosscorrelation normalization to reduce the number of detections to be further analyzed. The master waveform can be extracted from the data itself, selecting one of the strongest calls identified, or it could be a synthetic fin whale call. The method can use a number of classification parameters but the most effective are the ones that use the phase difference between the four channel recordings. These parameters can be used to identify and reject signals generated by postcritical incidence angle at the sea-floor.

\section{ACKNOWLEDGMENTS}

The Gulf of Cadiz data were collected for the NEAREST project, on behalf of the EU Specific Programme "Integrating and Strengthening the European Research Area," Sub-Priority 1.1.6.3, "Global Change and Ecosystems," Contract No. 037110. The Azores dataset was collected under the MoMAR project and provided to us by Wayne Crawford (Institut de Physique du Globe de Paris). One of the authors contribution (D.H.) was supported by the Office of Naval Research 1172 under the "Cheap DECAF" project, Grant No. 1173 N000141110615. We thank the editor and one anonymous reviewer for their constructive comments, which helped us to improve the manuscript. Publication supported by project FCT UID/GEO/50019/ 2013 - Instituto Dom Luiz.

Aki, K., and Richards, P. (1980). Quantitative Seismology, Theory and Methods (Freeman and Company, San Francisco, CA), Vol. 1, 557 pp.

Ambar, I., Serra, N., Neves, F., and Ferreira, T. (2008). "Observations of the Mediterranean undercurrent and eddies in the Gulf of Cadiz during 2001," J. Mar. Syst. 71, 195-220.

Arnulf, A. F., Singh, S. C., Harding, A. J., Kent, G. M., and Crawford, W. (2011). "Strong seismic heterogeneity in layer $2 \mathrm{~A}$ near hydrothermal vents at the Mid-Atlantic Ridge," Geophys. Res. Lett. 38, L13320, doi:10.1029/ 2011 GL047753.

Arnulf, A. F., Harding, A. J., Singh, S. C., Kent, G. M., and Crawford, W. (2012). "Fine-scale velocity structure of upper oceanic crust from full waveform inversion of downward continued seismic reflection data at the Lucky Strike Volcano, Mid-Atlantic Ridge," Geophys. Res. Lett. 39, L08303, doi:10.1029/2012GL051064.

Buckingham, M. J. (1998). "Theory of compressional and shear waves in fluid-like marine sediments," J. Acoust. Soc. Am. 103(1), 288-299.

Buckland, S. T., Anderson, D. R., Burnham, K. P., Laake, J. L., Borchers, D. L., and Thomas, L. (2001). Introduction to Distance Sampling (Oxford University Press, Oxford, UK), 432 pp.

Castagna, J. P. (1993). "AVO analysis-tutorial and review," in Offset-dependent Reflectivity-Theory and Practice of AVO Analysis, edited by J. Castagna and M. M. Backus (Society of Exploration Geophysicists, Tulsa, OK), pp. 3-37.

Chauhan, A., Rai, A., Singh, S. C., Crawford, W. C., Escartin, J., and Cannat, M. (2009). "OBS records of whale vocalizations from LuckyStrike segment of the Mid-Atlantic Ridge during 2007-2008," in Proceedings of the American Geophysical Union, Fall Meeting, San Francisco, CA, abstract \#S51D-07.

Corela, C. (2014). "Ocean bottom seismic noise: Application for the crust knowledge, interaction ocean-atmosphere and instrumental behaviour," Ph.D. thesis, University of Lisbon, 339 pp.

Crawford, W. (2013). (personal communication).

Crawford, W. C., Beguery, L., Daniel, R., Corela, C., Duarte, J. L., Hananto, N., Cordeiro, A., Pasquet, S., and Arnulf, A. (2008). BBMOMAR-2 cruise report, IPGP, $47 \mathrm{pp}$.

Crawford, W. C., Rai, A., Singh, S. C., Cannat, M., Javier Escartin, J., Haiyang Wang, H., Romuald, D. R., and Combier, V. (2013). "Hydrothermal seismicity beneath the summit of Lucky Strike volcano, Mid-Atlantic Ridge,” Earth Planetary Sci. Lett. 373, 118-128.

Crawford, W. C., and Singh, S. C. (2008). "Sediment shear properties from seafloor compliance measurements: Faroes-Shetland basin case study," Geophys. Prospecting 56(3), 313-325. 
Favretto-Cristini, N., Cristini, P., and de Bazelaire, E. (2007). "Influence of the interface Fresnel zone on the reflected P-wave amplitude modelling," Geophys. J. Int. 171(2), 841-846.

Hamilton, E. (1976). "Shear wave velocity versus depth in marine sediments: A review," Geophys. 41(5), 985-996.

Harris, D., Matias, L., Thomas, L., Harwood, J., and Geissler, W. H. (2013). "Applying distance sampling to fin whale calls recorded by single seismic instruments in the Northeast Atlantic," J. Acoust. Soc. Am. 134, 3522-3535.

Kasahara, J., Nagumo, S., Koresawa, S., Daikuhara, T., and Miyata, H. (1980). "Experimental results of vortex generation around ocean bottom seismograph due to bottom current," Bull. Earthquake Res. Inst., Univ. Tokyo 55, 169-182.

Lobue, N. (2012). (personal communication).

Marques, T. A., Thomas, L., Martin, S. W., Mellinger, D. K., Ward, J. A., Moretti, D. J., Harris, D., and Tyack, P. L. (2013). "Estimating animal population density using passive acoustics," Biol. Rev. 88(2), 287-309.

McDonald, M. A., Hildebrand, J. A., and Webb, S. C. (1995). "Blue and fin whales observed on a seafloor array in the Northeast Pacific," J. Acoust. Soc. Am. 98(2), 712-721.

Nafe, V. E., and Drake, C. L. (1963). "Physical properties of marine sediments," in The Sea, edited by M. N. Hill (Interscience, New York), Vol. 3, pp. 794-815.

NEAREST-Integrated Observations from Near Shore Sources of Tsunamis: Towards an Early Warning System (2012). http://nearest.bo. ismar.cnr.it (Last viewed March 16, 2014).

Nieukirk, S. L., Mellinger, D. K., Moore, S. E., Klinck, K., Dziak, R. P., and Goslin, J. (2012). "Sounds from airguns and fin whales recorded in the mid-Atlantic Ocean, 1999-2009," J. Acoust. Soc. Am. 131(2), 1102-1112.

Ottemöller, L., Voss, P., and Havskov, J. (2011). SEISAN: Earthquake analysis software for Windows, Solaris, Linux and Mac OSX, 361 pp.

Payne, R., and Webb, D. (1971). "Orientation by means of long range acoustic signaling in baleen whales," Ann. N.Y. Acad. Sci. 188, 110-141.

Rebull, O. G., Cusí, J. D., Fernández, M. R., and Muset, J. G. (2006).

"Tracking fin whale calls offshore the Galicia Margin, north east Atlantic ocean," J. Acoust. Soc. Am. 120(4), 2077-2085.

Roberts. R. G., Christoffersson, A., and Cassidy, F. (1989). "Real time events detection, phase identification and source location estimation using single station component seismic data and a small PC," Geophys. J. 97, 471-480.

Seher, T., Singh, S. C., Crawford, W. C., and Escartín, J. (2010). "Upper crustal velocity structure beneath the central Lucky Strike Segment from seismic refraction measurements," Geochem. Geophys. Geosyst. 11, Q05001, doi: 10.1029/2009gc002894.

Shipboard Scientific Party (1972). "Sites 135," in Initial Reports of the Deep Sea Drilling Project, Vol. 14, edited by D. E. Hayes, A. C. Pimm, J. P. Beckmann, W. E. Benson, W. H. Berger, P. H. Roth, P. R. Supko, and U. von Rad (U.S. Government Printing Office, Washington, DC), pp. $15-48$.

Širović, A., Hildebrand, J. A., and Wiggins, S. M. (2007). "Blue and fin whale call source levels and propagation range in the Southern Ocean," J. Acoust. Soc. Am. 122(2), 1208-1215.

Somoza, L., Anahnah, F., Bohoyo, F., González, J., Hernández, J., Iliev, I., León, R., Llave, E., Maduro, C., Martínez, S., Pérez, L. F., and Vázquez, T. (2007). "Informe científico-técnico, N/O L'ATALANTE" ("Scientific and technical report, R/V L'Atalante"), August 23 to September 9, 2007, project report, http://tierra.rediris.es/GulfofCadiz/GulfofCadiz_informe_final.pdf (Last viewed July 25, 2014)

Stafford, K. M., Citta, J. J., Moore, S. E., Daher, M. A., and George, J. E. (2009). "Environmental correlates of blue and fin whale call detections in the North Pacific Ocean from 1997 to 2002," Mar. Ecol. Prog. Ser. 395, 37-53.

Thompson, P. O., Findley, L. T., and Vidal, O. (1992). "20-Hz pulses and other vocalizations of fin whales, Balaenoptera physalus, in the Gulf of California, Mexico,” J. Acoust. Soc. Am. 92, 3051-3057.

Wang, R. (1999). "A simple orthonormalization method for stable and efficient computation of Green's functions," Bull. Seis. Soc. Am. 89(3), 733-741.

Watkins, W. A., Tyack, P., Moore, K. E., and Bird, J. E. (1987). "The 20-Hz signals of finback whales (Balaenoptera physalus)," J. Acoust. Soc. Am. 82, 1901-1912.

Webb, S. C. (1998). "Broadband seismology and noise under the ocean," Rev. Geophys. 36(1), 105-142, doi:10.1029/97RG02287.

Weirathmueller, M., and Wilcock, W. S. D. (2013). "An automatic single station multipath ranging technique for $20 \mathrm{~Hz}$ fin whale calls: Applications for ocean bottom seismometer data in the northeast Pacific," J. Acoust. Soc. Am. 134, 4147.

Weirathmueller, M., Wilcock, W. S. D., and Soule, D. C. (2013). "Source levels of fin whale $20 \mathrm{~Hz}$ pulses measured in the Northeast Pacific Ocean," J. Acoust. Soc. Am. 133, 741-749.

Wilcock, W. S. D. (2012). "Tracking fin whales in the northeast Pacific Ocean with a seafloor seismic network," J. Acoust. Soc. Am. 132(4), 2408-2419. 